\title{
WestVirginiaUniversity
}

THE RESEARCH REPOSITORY @ WVU

Graduate Theses, Dissertations, and Problem Reports

2020

\section{Frontal Brain Injury: Effects on Flexibility, Impulse Control, and Attention}

Christopher Matthew O'Hearn

WVU, cmohearn@mix.wvu.edu

Follow this and additional works at: https://researchrepository.wvu.edu/etd

Part of the Animal Experimentation and Research Commons, Behavior and Behavior Mechanisms Commons, and the Biological Psychology Commons

\section{Recommended Citation}

O'Hearn, Christopher Matthew, "Frontal Brain Injury: Effects on Flexibility, Impulse Control, and Attention" (2020). Graduate Theses, Dissertations, and Problem Reports. 7598.

https://researchrepository.wvu.edu/etd/7598

This Thesis is protected by copyright and/or related rights. It has been brought to you by the The Research Repository @ WVU with permission from the rights-holder(s). You are free to use this Thesis in any way that is permitted by the copyright and related rights legislation that applies to your use. For other uses you must obtain permission from the rights-holder(s) directly, unless additional rights are indicated by a Creative Commons license in the record and/ or on the work itself. This Thesis has been accepted for inclusion in WVU Graduate Theses, Dissertations, and Problem Reports collection by an authorized administrator of The Research Repository @ WVU. For more information, please contact researchrepository@mail.wvu.edu. 
Graduate Theses, Dissertations, and Problem Reports

2020

Frontal Brain Injury: Effects on Flexibility, Impulse Control, and Attention

Christopher Matthew O'Hearn

Follow this and additional works at: https://researchrepository.wvu.edu/etd

Part of the Animal Experimentation and Research Commons, Behavior and Behavior Mechanisms Commons, and the Biological Psychology Commons 
Frontal Brain Injury: Effects on Flexibility, Impulse Control, and Attention

Christopher M. O'Hearn

Thesis Submitted to the

Eberly College of Arts and Sciences at West Virginia University

in partial fulfillment of the requirements for the degree of

Master of Science in

Psychology

Cole Vonder Haar, Ph.D., Chair

Melissa Blank, Ph.D.

Karen Anderson Ph.D.

Department of Psychology

Morgantown, West Virginia

2020

Keywords: traumatic brain injury; flexibility; impulse control; attention Copyright 2020 Christopher O'Hearn 


\begin{abstract}
Frontal Brain Injury: Effects on Flexibility, Impulse Control, and Attention
\end{abstract}

Christopher M. O’Hearn

Traumatic Brain Injury (TBI) is defined as an impact to the head, penetration of the skull, or rapid deceleration of the skull, resulting in an alteration of brain function or neurological deficit. Cognitive deficits are common following TBI and often go unresolved due to a lack of effective treatments. These deficits often perseverate into the chronic post injury phase, so the development of rehabilitative strategies is imperative. Behavioral flexibility, impulse control, and attention are a few cognitive processes that are commonly affected by TBI. The current research compares these processes between rats with and without a severe frontal brain injury (TBI vs. Sham). Behavioral flexibility was measured with the attentional set shifting task (AST) and probabilistic reversal learning $(\mathrm{PbR})$. Differential reinforcement of low rate behavior (DRL) was used to measure impulse control. Cues associated with correct responding were used compare attention between TBI and Sham rats. The cues also served as an environmental treatment for TBI related deficits. Behavioral flexibility, measured by AST performance, was not affected by TBI, however TBI rats were impaired relative to Sham rats on PbR. Sham rats performed better on DRL when compared to TBI rats, suggesting that impulse control was impaired by frontal TBI. The cue treatment improved performance for TBI and Sham rats on both PbR and DRL. On PbR, cues improved TBI performance to Sham levels. Cues also improved TBI performance on DRL, but not to Sham levels. These data suggest that frontal TBI impairs impulse control and behavioral flexibility. The improvement seen in TBI rats associated with the cue treatment suggest that attention may somewhat intact following a brain injury. In addition, the differential improvement between PbR and DRL performance suggests that TBI related deficits in impulse control may be more difficult to treat than deficits in behavioral flexibility. 


\section{Acknowledgments}

I would like to thank Cole Vonder Haar and the Southern Regional Education Board for funding this research. I would especially like to thank Cole Vonder Haar for taking me as his first student and serving as my research mentor. His patience, flexibility, and generosity have allowed me to successfully complete this research. Thank you to my lab mates, Michelle Frankot and Kristen Pechacek, for assisting with my research, being my support system, and being my friends; together, we have developed a healthy and productive lab environment. Thank you to the various undergraduates who contributed to this project; their efforts and reliability are essential to the successful conduction of scientific research in our lab. Finally, I would like to thank the members of my committee, Cole Vonder Haar, Melissa Blank, and Karen Anderson for taking to time to review my document, participate in my oral defense, and provide feedback. 


\section{Table of Contents}

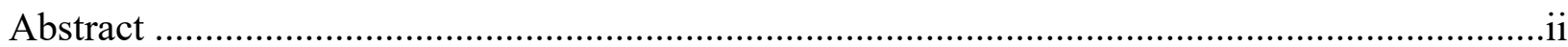

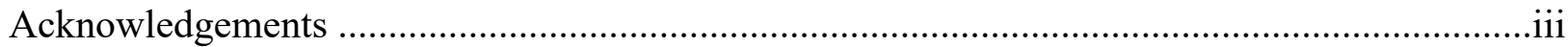

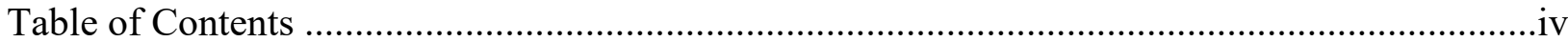

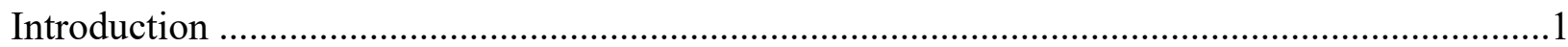

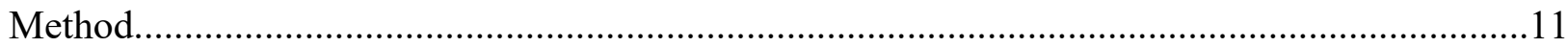

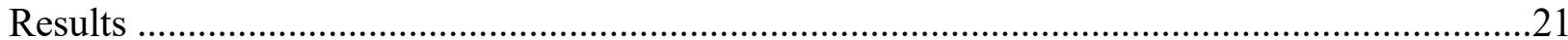

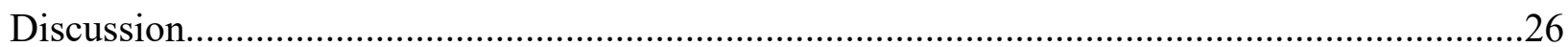

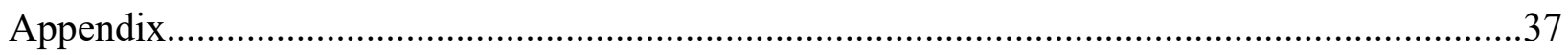

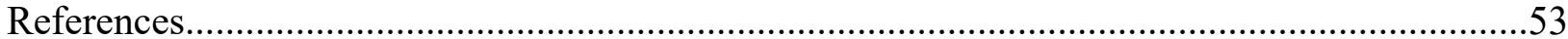




\section{INTRODUCTION}

\section{Traumatic Brain Injury}

In 2013, the Center for Disease Control (CDC) reported a total of 2.8 million traumatic brain injures (TBIs) in the United States, including hospitalizations, emergency room visits, and deaths. Populations most often affected by TBI include the elderly, athletes, and military personnel (Faul \& Coronado, 2015), and it is estimated that approximately 5.3 million Americans are currently living with a TBI-related disability (CDC, 2019). The direct and indirect financial expenses associated with TBI in 2010 were estimated at $\$ 76.5$ billion, and although severe TBIs account for the minority of total TBI incidences, they are responsible for $90 \%$ of those costs (Nguyen, 2016).

TBI is defined as an impact to the head, penetration of the skull, or rapid deceleration of the skull, resulting in an alteration of brain function or neurological deficit (Bayly et al., 2005; Menon, Schwab, Wright, \& Maas, 2010). There are a number of symptoms associated with TBI, including impaired cognitive function, motor function, sensation, and emotion regulation. These impairments may be acute or chronic and can have detrimental effects on interpersonal relationships, work life, self-care, daily living, and overall well-being (CDC, 2019). The Glasgow Coma Scale (GCS) is the most common measure of TBI severity (Teasdale \& Jennet, 1974). Using this scale, acute severity is scored as mild, moderate, or severe, and has a direct impact on functional outcomes up to 10 years after the initial injury (Ponsford, Draper, \& Schonberger, 2008). Lesion location (e.g. frontal lobe vs. temporal lobe) is also a primary determinant of associated functional deficits and symptoms (Stuss et al., 2000). 
Hospital protocols for TBI-related emergency department visits focus on controlling bleeding and maintaining stable intracranial pressure (ICP). Osmotherapy and/or decompression by means of craniotomy are the most common intensive care management strategies, however, both procedures are somewhat controversial due to associated risk factors and questionable efficacy (Maas, Stocchetti, \& Bullock, 2008). Of the 2.8 million annual TBIs in the United States, only 50,000 (1.7\%) result in death (CDC, 2019), so the development and implementation of effective therapeutics is a necessity. Post-injury rehabilitative strategies are centered around the improvement of motor function and other physical impairments (e.g. physical therapy/occupational therapy). However, cognitive and emotional deficits often go unresolved due to a lack of effective treatment and an inadequate understanding of the physiological and behavioral mechanisms by which these deficits occur (Maas et al., 2008).

\section{Chronic Functional Impairment}

Sensory and motor deficits are often related to damage in the somatosensory cortex or motor cortex, respectively, and may reduce coordination, movement, and balance (Miremami, Talauliker, Harrison, \& Lifshitz, 2014; Schönfeld et al., 2017). Motor dysfunction often persists chronically and may result in permanent physical disability (Walker \& Pickett, 2007). This is an example of overt chronic physical impairment associated with brain injury; more covert dysfunction may also persist into the chronic post injury phase. It is likely that even individuals who do not appear to demonstrate overt impairments during follow-up assessments still experience some deficit relative to pre-injury motor function (Walker \& Pickett, 2007). When lesions were inflicted to the forelimb area of the motor cortex in rats, no deficits were shown in gait or gross motor function. However, persistent deficits in fine motor function and sensation were detected (Schönfeld et al., 2017). These studies demonstrate one of the major hurdles 
associated with the examination and treatment of chronic TBI-induced dysfunction: detection of subtle chronic deficits.

Emotional dysregulation related to depression, aggression, affect change, or anxiety is also common following TBI (Bombardier et al., 2010; Hoofien, Vakil, \& Donovick, 2001), and may impose long-term functional consequences as well (Ponsford, et al., 2008). Alterations in emotion regulation are usually associated with damage in the frontal lobe of the brain (Green, Turner, \& Thompson, 2002; Paradiso, Chemerinski, Yazici, Tartaro, \& Robinson, 1999). The prevalence of major depressive disorder (MDD) in hospitalized TBI patients 12 months postinjury is $53.1 \%$, which is 7.9 times greater than that of individuals with orthopedic injury (6.7\%) (Bombardier et al., 2010). Impact to the medial frontal cortex increases depressive-like behavior in rats on the forced swim test (FST) and sucrose preference test (SP) during the acute postinjury phase (Moritz, Geeck, Underly, Searles, \& Smith, 2014). However, these behaviors are generally not tested using repeated measures or during the chronic post injury phase, and unpublished data from the Vonder Haar lab detected no differences in performance between TBI and control rats during chronic repeated exposure to the FST or SP test. These unpublished results demonstrate once again the difficulty that may be encountered when attempting to detect chronic deficits.

Cognitive deficits are particularly problematic; they are debilitating in and of themselves, but their effects may be two-fold. Research suggests that they may mediate other deficits like emotion dysregulation (Ponsford et al., 2008) Cognitive deficits are common following TBI; these are usually associated with damage to the frontal lobe (Lindner et al., 1998; Vonder Haar \& Winstanley, 2016). The detrimental nature of cognitive dysfunction in addition to its mediating effects on other deficits suggest that effective cognitive therapeutics may substantially augment 
recovery. Cognitive deficits refer to impairments in executive functions (e.g. behavioral flexibility, impulse control), information processing, memory, attention, or learning (Whitnall, McMillan, Murray, \& Teasdale, 2006). Much like sensory-motor deficits and emotional deficits, cognitive deficits associated with severe TBI often difficult to detect during the chronic postinjury phase (Soldatovic-Stajic et al., 2014). The current research examined chronic impairment to three cognitive processes (behavioral flexibility, impulse control, and attention) in rodents and attempted to improve outcomes using an environmental manipulation.

\section{Chronic Cognitive Dysfunction}

Behavioral flexibility (or cognitive flexibility) refers to the pattern of behavior involved with strategically adapting to environmental changes (Butts, Floresco, \& Phillips, 2013). This type of behavior is highly associated with the medial prefrontal cortex (mPFC) and orbitofrontal cortex (OFC) of rats (Butts et al., 2013). Impulse control refers to the ability of an organism to control, inhibit, or suppress behavior (Kocka \& Gagnon, 2014). Deficits in impulse control may result from injury to the PFC and contribute to the occurrence of inopportune or inappropriate behavior (Menon, Adleman, White, Glover, \& Reiss, 2001). Attention refers to behavior associated with detecting cues and other stimuli in the environment (Braun et al., 1989). PostTBI attentional deficits are likely associated with the dysfunction in the anterior cingulate cortex (Kim, Wasserman, Castro, \& Freeman, 2016) and mPFC (Passetti, Chadasuma, \& Robbins, 2002).

Humans exist in a complex/dynamic environment, such that flexibility, impulse control, and attention are necessary for engagement in daily life, work life, and interpersonal relationships. The dysregulation of any of these cognitive processes may have detrimental effects on functional recovery and reintegration following a brain injury. Unfortunately, these deficits 
often persist chronically in TBI patients due to ineffective therapeutics or a complete absence of treatment (Maas et al., 2008). The inadequacy of treatment strategies is likely due to the currently limited understanding of the behavioral and physiological mechanisms by which these cognitive deficits occur (Maas et al., 2008). Due to the heterogeneity of clinical TBI, preclinical animal models are an imperative part of the TBI research effort. Animal models are used to simulate the cognitive-behavioral deficits seen in human TBI populations. Animal research uses a combination of different injury models and behavioral tests to better understand the relationship between brain injury and behavior (Bondi et al., 2015). However, when using animal models, it important to consider generalizability, potential confounds, and to recognize the relative limitations of translational animal research.

\section{Behavioral Flexibility}

In humans, this type of behavior can be tested using the Wisconsin Card Sorting Task (WCST), which requires participants to shift between sorting rules (Milner, 1963). The Attentional set-shifting task (AST) was adapted from the WCST for use in preclinical research (Diaz, Robbins, \& Roberts, 1996). Damage to the frontal lobe reduces optimal performance on the WCST and AST for humans and preclinical models, respectively (Diaz, Robbins, \& Roberts, 1996; Levin et al., 1993). The AST uses compound stimuli to examine behavioral flexibility in animals. It is a two-phase task that requires the animal to learn a particular stimulus-reinforcer relation during the first "set" phase of the task, then requires an extradimensional shift to a new stimulus during the "shift" phase (e.g. light position to lever position) while the original stimulus is still present (Butts et al., 2013). Historically, the AST has used different digging mediums (e.g. dirt, paper, sand) paired with olfactory, visual or auditory cues to signal reinforcer availability (Tait, Chase, \& Brown, 2014). The task has since been adapted for use in operant chambers 
using two levers (left/right) and two stimulus lights (left/right). Set-shifting behavior is primarily mediated by the PFC (Butts et al., 2013). Behavioral flexibility in the AST is quantified by examining the number of perseverative responses observed during the shift-phase (e.g. responding that meets criteria for the original set-phase contingency), and number of trials required to meet criteria on the new discrimination.

Reversal leaning (RL) is also a two-phase task conducted in an operant chamber that requires the animal to learn a response-reinforcer relation during the first phase. Similar to the AST, the second phase requires a shift in stimulus attention, but during the "reversal" phase of $\mathrm{RL}$, the shift is intradimensional (e.g. left lever to right lever). This type of RL can be examined individually or used as part of the previously described AST (Cox, Cope, Parsegian, Floresco, Jones, \& See, 2016). This type of RL is mediated by the OFC and the dorsomedial striatum; perseverative responding is used to quantify flexibility (Butts et al., 2013; Dalton, Phillips, \& Floresco, 2014).

Probabilistic reversal learning $(\mathrm{PbR})$ is a variation of $\mathrm{RL}$ that is also conducted in an operant chamber (Dalton et al., 2014). This task associates high-probability reinforcement (e.g. $80 \%$ ) with the "correct" lever, and a low-probability reinforcement (e.g. 20\%) with the "incorrect" lever. Once a predetermined number of responses occur on the "correct" lever, the probabilities are reversed. Number of reversals per session is used to quantify flexibility in this task. The probabilistic nature and decreased discriminability due to unreinforced correct responses associated with $\mathrm{PbR}$ requires the activation of different brain regions from the "all or none" RL procedure. PbR is highly dependent on function in the nucleus accumbens (NAc) (Dalton et al., 2014) and OFC (Amodeo, McMurray, \& Foitman, 2017), and somewhat dependent on the PFC (Dalton et al., 2016). 


\section{Impulse Control}

Impulsivity can be associated with either decision-making or motor function (Diergaarde, 2008). In humans, this type of behavior can be measured using the go/no-go (GNG) task, in which an individual is directed to respond to one stimulus (e.g. red) and inhibit responding to another stimulus (e.g. blue) (Braun, Daigneault, \& Champagne, 1989). Impulse control in animals can be examined in several different tasks, however, due to its simplicity, differential reinforcement of low rate behavior (DRL) is one of the most direct measures of motor impulsivity. In DRL, animals must meet a particular inter-response time (IRT) criteria between two consecutive responses in order to receive a reinforcer (Wilson \& Keller, 1953). For example, in a DRL 20-s schedule, an initial response occurs, starting a 20-s timer. If the next response occurs before $20 \mathrm{~s}$ elapses, the timer restarts. However, if the next response occurs after $20 \mathrm{~s}$ has elapsed, a reinforcer is delivered (Wilson \& Keller, 1953). Dopamine depletion in the PFC is associated with imparied performance on DRL (Sokolowski \& Salamone, 1994); number of earned in a DRL session is positively correlated with impulse control. It should be noted that dopamine levels in the PFC may be positively correlated with the release of dopamine in the NAc, as previous research has demonstrated that stimulation of the PFC resulted in the phasic release of dopamine in the NAc (Hill et al., 2018).

\section{Attention}

For animals and humans alike, behavioral tasks require the organism to exert some level of attention, which should be considered when characterizing post-TBI cognitive deficits. AST, $\mathrm{RL}, \mathrm{PbR}$, and $\mathrm{DRL}$ all require the animal to attend to a stimulus (e.g. lever side, stimulus light, time elapsed) in order to achieve optimal performance. Attention is a complex operation that requires the integrated function of several different brain regions including the ACC (Kim et al., 
2016) and mPFC (Passetti, Chadasuma, \& Robbins, 2002). The overlap in associated brain areas between impulse control, behavioral flexibility, and attention creates a potential confound in each of these tasks, such that low performance may be accounted for by difficulty attending to the more subtle stimuli associated with each behavioral test (e.g. probability, time), rather than impulse control or behavioral flexibility. However, deficits associated with attention can be reduced using more salient cues to signal reinforcer availability (Ellen \& Butter, 1969; Farina et al., 2015), so salient cues were used in the current research to account for deficits in attention

\section{Models of Brain Injury}

The heterogeneity of TBI makes it difficult to study in human populations. Animal models facilitate the experimental examination of TBI by providing control over lesion severity, location, and impact type. Concussion or mild TBI (mTBI) is characterized by transient neurological abnormalities following trauma to the head. Symptoms include headache, fatigue, dizziness, emotion dysregulation, and memory impairment (Junn, Bell, Shenouda, \& Hoffman, 2015). When studying non-penetrative mild TBI, a closed head injury model is often used. The closed head impact model of engineered rotational acceleration (CHIMERA) uses a piston, accelerated by air pressure, to impact the top of the skull. The CHIMERA model allows for adjustment of impact force, repeated injuries, and simulates the rotational force that is often associated with concussive injuries (Namjoshi et al., 2014). The blast overpressure model of TBI was developed to better understand injuries sustained by military personnel and others affected by war (Long, Bentley, Wessner, Cerone, Sweeney, \& Bauman, 2009). It simulates the type of injury an individual may sustain when exposed to an improvised explosive device (IED) (Ahlers et al., 2013). This model induces a brain injury by funneling pressure from gas or explosives to an anesthetized animal (Cernak \& Noble-Haeusslein, 2010). 
Focal TBI models have been developed to provide a high level of specificity regarding severity and lesion location, with the intention of replicating deficits associated with closed-head TBI in specified brain areas. Focal TBI can be studied using controlled cortical impact (CCI), fluid percussion injury (FPI), or weight drop (WD) methods. The WD method is a closed-head model in which a tube-guided weight is dropped directly on the skull of the animal. Severity is manipulated by adjusting the height of the drop and mass of the weight (Feeney, Boyeson, Linn, Murray, \& Dail, 1981). However, potential skull fracture, secondary injury, and relative imprecision limit the utility of the WD model (Rostami 2012). The FPI is an open-head (craniotomy) model that uses fluid pressure to induce an injury directly to the cortex (McIntosh, Nobel, Andrews, \& Faden, 1987). The FPI model provides a high level of precision over injury location, but high severity injuries are difficult to reproduce due to the risk of mortality resulting from distal damage (Morales et al., 2005). Thus, the CCI model is often considered the superior focal model because it provides control over location, depth, velocity, and dwell time. It is an open-head model that induces a lesion by applying direct mechanical force to the cortex using a steel piston (Lighthall, 1988). High levels of severity can be examined using the CCI model because damage from initial impact is localized to the injury site (Lighthall, 1988). The CCI model was the used in the current research to induce an injury to the PFC with little distal damage. The specificity of the injury decreased the likelihood of confounding cognitive dysfunction with unrelated deficits (e.g. motor impairment).

\section{Experimental Design}

\section{Flexibility vs. Attention}

The first goal of the current study was to determine the degree of deficit in behavioral flexibility after inducing a severe frontal TBI (CCI model) in rats (Experiment 1a and 1b), and to 
identify the role of attention in those deficits (Experiment $1 b$ ). It was hypothesized that behavioral flexibility would be impaired following severe TBI, indicated by decreased performance on $\mathrm{AST}$ and $\mathrm{PbR}$. In addition, it was expected that impaired attention would contribute to deficits in behavioral flexibility despite the introduction of salient cues associated with "correct" responding on $\mathrm{PbR}$.

\section{Impulse Control vs. Attention}

The second goal of the current study was to characterize impulse control following severe TBI in rats and dissociate degraded impulse control from attentional deficits (Experiment 2). It was hypothesized that impulse control would be impaired following severe TBI, indicated by fewer correct responses on DRL. Similar to deficits in behavioral flexibility, it was expected that post-TBI attentional deficits would contribute to deficits in impulse control. It was hypothesized that TBI performance on DRL would be rescued using salient cues to signal reinforcer availability.

\section{METHODS}

\section{Animals}

Male Long-Evans rats maintained at approximately $85 \%$ ad libitum weight using grainblend chow were used in the following experiments. Prior to surgery rats were pair-housed in a vivarium in standard-ventilated cages. Due to post-injury aggression, rats were pair housed in ventilated Opti-cages (Animal Care Systems, Colorado) with a divider in the middle. Rats were maintained on an 12:12-hr light/dark cycle and had have continuous access to water in their home cages. All behavioral sessions occurred at approximately the same time each day during the rat's active dark cycle. Post-session feedings occurred after completing behavioral tasks; rats were weighed once per week. 


\section{Testing Apparatus}

All behavioral testing and training sessions occurred in an isolated bank of 16 standardoperant chambers with dimensions of $30 \times 24 \times 21 \mathrm{~cm}$ (Med Associates, St. Albans, Vermont). Each chamber was enclosed in a sound-attenuating crate and the bank was illuminated with red light. White noise generators were used to mitigate the effects of extraneous sounds on behavior. The right panel of each chamber was equipped with a food hopper and two retractable levers, one to the left of the hopper, and one to the right. A white stimulus-light was positioned above each lever and a house-light was located at the top of each chamber. The left panel of each chamber was equipped with 5 nose-poke holes; however, they were not in operation during this experiment. Two DIG-716B boards interfaced the chambers to a pair of computers equipped with MED PC data collection software (Med Associates, St. Albans, Vermont).

\section{Surgery}

Surgical procedures were performed using methods adopted from previous studies (Hoane, Akstulewicz, \& Toppen, 2003; Vonder Haar, Anderson, \& Hoane, 2011). To mitigate potential infection, aseptic techniques were employed. Ketoprofen $(5 \mathrm{mg} / \mathrm{kg})$ were administered subcutaneously before surgery for analgesia. Bupivacaine $(0.1 \mathrm{~mL})$ was used as a local anesthetic. Each rat was fully anesthetized using a combination of oxygen $(0.5 \mathrm{~L} / \mathrm{min})$ and isoflurane (2-4\%), and subsequently placed in a stereotaxic apparatus. A heating pad was used to regulate body temperature while under anesthesia. Once the nociceptive flexion reflex could no longer be detected using the toe-pinch method, a $2.0 \mathrm{~cm}$ incision was made along the midline revealing the top of skull. TBI group rats received a craniotomy, $6.0 \mathrm{~mm}$ in diameter, over the medial prefrontal cortex $(\mathrm{AP}+3.0, \mathrm{ML}+0.0$ from bregma) using a micro-drill. A stainless-steel circular impactor tip (5.0 mm diameter) was positioned over the craniotomy and an 
electromagnetic impactor device (Leica Biosystems, Buffalo Grove, IL) was used to drive the impactor tip into the cortex to a depth of $2.5 \mathrm{~mm}$, at a rate of $3 \mathrm{~m} / \mathrm{s}$. The impactor tip remained in the cortex for $500 \mathrm{~ms}$ before it was retracted. Once post-injury bleeding was attenuated, the incision was sutured, and the rat was placed in a heated recovery chamber until it regained consciousness. Sham rats underwent a similar surgical procedure, except that they did not receive a craniotomy or a brain injury (Cole et al., 2011; Martens, Vonder Haar, Hutsell \& Hoane, 2012). During a 1-week post-surgical recovery period, animals were examined daily to assess pain signs, activity level, hydration, weight fluctuations, and infection. Additional care was administered as needed.

\section{Experiment 1a: Attentional Set Shifting}

Experimentally-naïve Long Evans rats $(\mathrm{N}=40)$ as described above, randomly assigned to Sham $(n=21)$ and TBI $(n=19)$ groups, were used in this experiment. To mitigate stress associated with experimenter handling, rats were handled $3 \mathrm{~min}$ per day, for three days prior to surgery. One day before behavioral testing began, 25 sucrose pellets were dropped into the home cage of each rat to eliminate neophobia. Behavioral training and testing procedures were adapted from previous studies (Butts et al., 2013; Brady \& Floresco, 2015; Cox et al., 2016). Phase 1 ('set'), the cue discrimination task, reinforced responses corresponding to the location of the stimulus light (left/right). Phase 2 ('shift'), the response discrimination task, reinforced responses to one side (left/right), regardless of light position. Phase 3 ('reversal'), the response reversal task, reinforced responses to the side opposite the previous phase (e.g. left to right).

\section{Lever Press Training}

In order to examine chronic deficits, chamber habituation and hopper training began three weeks after surgery and consisted of manually dispensing 10 pellets into the food hopper, then 
placing the rat into the operant chamber with all outputs turned off. The rat remained in the chamber for 30-min to explore and consume the pellets. Hopper training sessions continued for two days or until all 10 pellets were consumed during the allotted time.

Lever-pressing behavior was shaped using a two lever autoshaping procedure (Brown \& Jenkins, 1968). During autoshaping sessions, a pellet was delivered every $35 \mathrm{~s}$ on average (VT35). Ten seconds before the pellet was delivered both levers were extended. If the rat pressed either lever before $10 \mathrm{~s}$ elapsed the lever retracted, and a pellet was delivered immediately. All pellet deliveries were paired with the illumination of the hopper-light. Autoshaping sessions lasted 60 min and a maximum of 100 pellets were available. Sessions continued until 40 lever presses occurred between the two levers. If lever-pressing did not occur within three sessions, lever-pressing was hand shaped (Ferster \& Skinner, 1957).

\section{Retractable Lever Press Training}

Retractable lever press training began the next day, following autoshaping. Each lever was presented 45 times in pseudorandom order, such that neither lever was presented more than twice in a row. At the start of each trial, the house light was illuminated, and the lever was extended for $10 \mathrm{sec}$. If a response occurred, the lever was retracted immediately, the hopper light illuminated, the house light was extinguished, and a pellet was delivered. If the rat did not respond the lever was retracted, the house light was extinguished, and an omission was recorded. A 10-s ITI was in effect. Sessions lasted 30 min and continued until fewer than five omissions occurred for two consecutive days.

\section{Lever Preference Assessment}

The preference assessment consisted of seven blocks, each with up to eight trials. During the first trial of each block, both levers were presented to the rat simultaneously for $10 \mathrm{~s}$. The 
first press on a lever distinguished it as the initial lever and a reinforcer was delivered as described above. If the subsequent response was on the lever opposite the initial lever, a reinforcer was delivered, and the rat moved on to the next block of trials. However, if the subsequent response was on the initial lever, no reinforcer was delivered, and the next trial of the same block was initiated. If the rat responded on the initial lever 7 consecutive times in the same block, the initial lever was retracted during trial 8 so that only a response on the opposite lever could occur. Once a response on the opposite lever occurred the next block began. The lever with the most initial responses was recorded as the rat's preference. These data were used to control for the effects of side preference during behavioral testing; preference data was used to determine the "correct lever" for each animal during the shift and reversal phases of AST.

\section{Phase 1 (Set): Cue Discrimination Task}

The cue discrimination task is considered the "set" task in this sequence because it sets the initial response requirement. Prior to the start of a trial the chamber was dark and both levers were retracted. The initiation of a trial was marked by the illumination either the left or right stimulus-light. After $3 \mathrm{~s}$, both the left and right levers were extended, and the rat was allotted 10 $\mathrm{s}$ to make a response. During this phase, a correct response was a press to the lever that corresponds to the stimulus light (e.g. left stimulus light illuminated + press to left lever $=$ correct response). If a correct response was made both levers were retracted, the hopper-light was illuminated, and a sucrose pellet was delivered. If an incorrect response was made, both levers were retracted, and no pellet was delivered. If no response occurred within $10 \mathrm{~s}$ of the initial lever extension the trial was recorded as an omission and the lever retracted. Stimuluslight presentations occurred pseudo-randomly such that neither light was illuminated for more than two consecutive trials. The task continued for a maximum of three days, with 200 trials per 
day, until 10 consecutive correct responses occurred. If an animal did not meet criterion within 3 days, it was removed from the experiment. An incorrect response disrupted a chain of consecutive correct responses, however omissions did not. If criterion was met on the first day, a minimum of 30 trials must have occurred before the task was completed. Trials to criterion, errors to criterion, and omissions were the primary dependent measures during this phase. The response discrimination task began the next day after criterion was met.

\section{Phase 2 (Shift): Response Discrimination Task}

The response discrimination task is considered the "shift" task in this sequence because it requires an extradimensional shift away from the stimulus light and towards one of the levers. In this condition the first session started with 20 "reminder" trials that were identical to the cue discrimination task Phase 1, after which a shift to the response discrimination task occurred. During response discrimination, a response on the lever opposite of the rat's recorded preference was considered correct, regardless of the position of the stimulus-light. A response on the lever associated with the rat's initial preference was now considered incorrect and was recorded as an error. All other conditions were identical to the cue discrimination task. The stimulus lights continued to alternatively illuminate as they did in the previous condition, however they were no longer associated with the reinforcement contingency, and were considered a distractor. Trials to criterion, errors to criterion, and omissions were the primary dependent measures. The response reversal task started the following day once this task was complete.

\section{Phase 3 (Reversal): Response Reversal Task}

The first session of this condition started with 20 reminder trials that employed the contingency from the previous phase in which only responses to the lever opposite of the rat's preference were reinforced. The reversal was then introduced and only responses on the rat's 
initial preference lever were reinforced. All other conditions were identical to the previous phase. Once again trials to criterion, errors to criterion, and omissions were the primary dependent measures.

\section{Error Analysis}

Phase 2 (shift) errors. For the Phase 2 error analysis, sessions were divided into 16 trial blocks (9 per session). Errors in Phase 2 were divided into two categories: perseverative or regressive. These categories determined if an error was due to an inability to shift away from the previous contingency (perseverative) or if they were related to deficits in learning and maintaining the new contingency (regressive or never reinforced). Perseverative errors were those made to the incorrect lever when the stimulus light was illuminated over it. Regressive errors were also made on the incorrect lever when the stimulus light was illuminated over it, but errors were only considered regressive once less than 6 occurred during a single 16-trial block (only accounts for errors associated with the stimulus light).

Phase 3 (reversal) errors. Once again, each session was divided into nine 16-trial blocks. Perseverative and regressive errors now occurred on the non-reinforced lever. All other criteria were the same. Figure 1 is a visual representation of these error analyses.

\section{Experiment 1b: Probabilistic Reversal Learning}

The methods proposed in Experiment $1 \mathrm{~b}$ were adapted from the $\mathrm{PbR}$ methods used by Dalton, Philips, and Floresco (2014). Training began seven days after the completion of Experiment 1a and used the same rats. Rats were already separated into Sham and TBI groups, and with the addition of a cue variable, were further separated (based on performance) into Sham $(n=11)$, TBI $(n=10)$, Sham-Cue $(n=10)$, and TBI-Cue $(n=9)$ groups. Testing continued for five weeks. 


\section{Probabilistic Reversal Learning}

The PbR task consisted of 200 discrete trials separated by a 15-s inter-trial interval. Trials were preceded by a dark chamber with both levers retracted. The initiation of a trial was distinguished by presentation of both levers. During the first trial, differential probabilities of reinforcement were assigned to each lever ("correct" lever $=80 \%$, "incorrect" lever $=20 \%$ ). After the levers were presented, $10 \mathrm{~s}$ was allotted for a response to occur. If eight consecutive choices on the "correct" lever occurred (regardless of if they were reinforced), the probabilities associated with each lever were reversed. Each time the levers are reversed, it is counted as a "reversal," which is the primary measurement of flexibility associated with this task. Reinforced choices on both the "correct" and "incorrect" lever caused the retraction both levers, illumination the hopper-light, and delivery of a sucrose pellet. Non-reinforced choices from both the "correct" and "incorrect" lever were followed by only the retraction of both levers. If no response occurred within $10 \mathrm{~s}$ both levers were retracted, and the trial was recorded as an omission. Omissions did not disrupt consecutive correct choices. Win-stay and lose-shift responses were also recorded. A win-stay response was recorded when a rat received a reinforcer on one trial, and then chose the same lever on the next trial. Lose-shift responses were the opposite; such that a response was considered lose-shift if a reinforcer was not delivered following a lever press, and the rat chose the alternative lever on the next trial. For the cue groups, a stimulus-light was illuminated over the correct lever at the $3 \mathrm{~s}$ before each trial started and remained illuminated until the trial was completed. No stimulus-light was illuminated at any point during sessions for the No-Cue groups. Variables of interest were number of reversals, omissions, win-stay responses, and loseshift responses.

\section{Experiment 2: Differential Reinforcement of Low Rate Behavior}


DRL (Wilson \& Keller, 1953) performance was assessed in experimentally-naïve Long Evans rats $(\mathrm{N}=38)$, as previously described. This experiment used a 2 x 2 design with rats separated into the following groups: Sham $(n=11)$, TBI $(n=8)$, Sham-Cue $(n=9)$, and TBI-Cue $(n=10)$. In order to examine chronic deficits, surgeries occurred 3 weeks prior to behavioral training. To mitigate stress associated with experimenter handling, rats were handled 3 min per day, for three days prior to surgery. Sucrose pellets were used as reinforcers in behavioral training/testing. One day before behavioral testing began, 25 sucrose pellets were dropped into the home cage of each rat to eliminate neophobia.

\section{Hopper \& Lever Press Training}

Hopper training and AutoShaping procedures in Experiment 2 were identical to the procedures used in Experiment 1, except that only one lever (counterbalanced left/right across subjects) was employed due to the single-lever nature of the DRL task.

Once AutoShaping was complete, a continuous-reinforcement schedule (FR1) was in effect such that one lever press resulted in the delivery of a reinforcer. FR1 sessions lasted 30 min and were in effect until 60 presses occurred for two consecutive days.

\section{DRL Testing}

Following FR1 training, a DRL 20-s schedule was in effect, such that a response initiated a 20-s timer. A reinforcer was delivered only if the next response occurred after the 20 -s timer had completed. Any response that occurred before the 20 -s timer had completed reset the timer (Costa, Bueno, \& Xavier, 2004; Numan, Seifert, \& Lubar, 1975). The stimulus light above the active lever was illuminated upon reinforcer availability for the Cue groups. All DRL sessions lasted $60 \mathrm{~min}$. Percent correct responses and IRT were the primary dependent measures. Testing continued for five weeks. 


\section{Histology}

When behavioral testing was completed animals were anesthetized with a lethal dose of pentobarbital and transcardially perfused. The brain was then removed from the skull and postfixed in $3.7 \%$ formaldehyde for $24 \mathrm{hr}$. Brains were then transferred to a $15 \%$ sucrose solution for two days, and then a 30\% sucrose solution until fully saturated ( 3 days minimum). Once fully saturated, the brains were embedded into gel blocks (15\% gelatin; 4-5 brains per block), frozen, and sliced on a microtome at $40 \mu \mathrm{m}$ along the coronal plane. Slices were then mounted on electrostatically-subbed slides for staining.

\section{Thionin Stain}

A Thionin stain was conducted to increase tissue visibility for lesion analysis. Slides were rehydrated using a series of washes administered in the respective order: Citrisolv ( 1 x 5 min $)$, $100 \%$ EtOH (2 x $2 \mathrm{~min}), 95 \%$, EtOH (1 x $2 \mathrm{~min}), 50 \%$, EtOH (1 x $2 \mathrm{~min}), 50 \%$ EtOH (1 x 1 $\min )$, and $\mathrm{dH}_{2} \mathrm{O}(1 \times 1 \mathrm{~min})$. They were then placed in a Thionin solution $(20 \mathrm{sec})$ for staining and dehydrated by reversing the previous sequence of washes. After dehydration, slides were then cover-slipped and allowed to dry overnight.

\section{Data Analysis}

\section{AST}

Repeated Measures ANOVAs were used to assess differences in trials to criterion (log), errors to criterion (log), omissions (inverse), perseverative errors (square root), and regressive errors $(\log )$ between Sham and TBI groups. A Box-Cox test was used to determine necessary transformations, and because there were two groups in this experiment, post-hoc analyses were conducted using a 2-sample t-test. The critical $p$-value for these analyses was set to $p<0.05$. 


\section{PbR}

The main effects of cue (cue/no cue), injury (TBI/Sham), and time (session) on number of reversals (log), omissions (inverse square root), win-stay responses (square), and lose-shift responses (square) were analyzed using a linear mixed-effects regression (LMER). A BoxCox test was used to determine necessary transformations. The critical $p$-value for these analyses was set to $p<0.05$.

\section{DRL}

The main effects of cue (cue/no cue), injury (TBI/Sham), and time (session) on percent correct responses (square root), IRT (log), and total responses (inverse square root) were analyzed using a multiple linear regression. A Box-Cox test was used to determine necessary transformations. The critical $p$-value for these analyses was set to $p<0.05$. Mean and standard deviation (SD) were calculated for IRT and a line graph was used to visually inspect IRT distributions $(\log$ IRT $\%>)$.

\section{Lesion Analysis}

Digital images (600dpi) of the brain slices, traversing the area of the lesion $(+5.0,+4.0$, $+3.0,+2.0,+1.0$ from bregma) (Paxinos \& Watson, 2009), were measured using ImageJ (NIH, Bethseda, MD). Lesion volume and the remaining brain volume was estimated (Vonder Haar, Anderson, \& Hoane, 2011), and a one-way ANOVA was used to compare brain volumes between TBI, Sham, TBI-Cue, and Sham-Cue rats (Lesion Volume $\sim$ Group). Four brains were selected pseudo-randomly (2 TBI, 2 Sham) from each experiment and analyzed by an alternate researcher to ensure accuracy. A 2-sample T-Test was used to examine differences between calculated brain volume between original measurements and inter-rater agreement (IRA) measurements. All researchers conducting lesion analyses were blinded to group assignment 
associated with each brain. The critical $p$-value for these analyses was set to $p<0.05$. Tukey's HSD was used for post-hoc analyses because there were four groups.

\section{RESULTS}

\section{Experiment 1a: Set-Shifting \& Response Reversal}

Trials to criterion, errors to criterion, and omissions in the set, shift, and response reversal phases of Experiment 1a were analyzed using a series of $2 \times 3$ repeated measures ANOVAs [Injury (Sham, TBI) X Phase (P1, P2, P3)]. For trials to criterion there was no Injury X Phase interaction $(F(1,117)=0.274, p=0.761)$. For errors to criterion there was no Injury X Phase interaction $(F(1,117)=0.153, p=0.850)$. See figures 2 and 3. There was a significant Injury $\mathrm{X}$ Phase interaction for omissions $(F(1,117)=4.185, p=0.013)$. Post-hoc analyses using 2-sample T-Tests revealed that TBI rats had significantly more omissions than Sham rats in Phase 3 $(t(18.96)=-2.18, p=0.042)$. See Figure 4

An error analysis was conducted to examine perseverative and regressive errors separately. A 2x2 repeated measures ANOVA [Injury (Sham, TBI) x Phase (P2, P3)] was used for each respective analysis. There were no significant between Injury X Phase interactions for perseverative errors $(F(1,78)=3.830, p=0.054)$, or regressive errors $(F(1,78)=0.809, p=$ 0.371). See Figure 5 and Figure 6.

\section{Experiment 1b: Probabilistic Reversal Learning}

Reversals for trials completed (accounting for omissions) on PbR were analyzed using LMER [Fixed: Injury (Sham, TBI) x Cue (Cue, No Cue) x Time, Random: Subject]. There was no omnibus effect of Injury $(F(1,76.02)=0.04, p=0.840)$. There was no omnibus effect of Cue $(F(1,76.02)=3.82, p=0.054)$. There was a significant omnibus effect of Time $(F(1,920.42)=$ $348.70, p<0.001)$ such that reversals increased over time for all rats $(\beta=0.37, t=13.268, p<$ 
0.001). There was a significant omnibus effect of Injury X Cue $(F(1,76.02)=9.83, p=0.002)$ such that the TBI-Cue group achieved more reversals than the Sham-Cue group $(\beta=0.36, t=$ 2.022, $p=0.046)$, and the Sham group achieved more reversals than the TBI group $(\beta=0.47, t=$ $-2.423, p=0.018)$. There was no omnibus effect of Injury X Time $(F(1,920.42)=0.03, p=$ 0.855). There was a significant omnibus effect of Cue X Time $(F(1,920.42)=149.55, p<0.001)$ such that Cue group rats exhibited greater increases in number of reversals achieved over time $(\beta$ $=0.25, t=6.536, p<0.001)$. There was a significant omnibus effect of Injury X Cue X Time $(F(1,920.42)=7.63, p=0.006)$, such that number of reversals for TBI-Cue rats increased more over time when compared to Sham-Cue rats, $(\beta=-0.08, t=-2.023, p=0.043)$. TBI and Sham group rats did not exhibit differential performance over time $(\beta=0.07, t=-1.881, p=0.060)$. These data are shown in Figure 7.

Omissions on PbR were examined using LMER [Fixed: Injury (Sham, TBI) x Cue (Cue, No Cue) x Time, Random: Subject]. There was a significant omnibus effect of Injury such that TBI rats omitted more than Sham rats overall $(F(1,48.98)=13.69, p<0.001)$. There was no omnibus effect of Cue $(F(1,48.98)=0.23, p=0.637)$. There was a significant omnibus effect of Time such that Omissions for all rats decreased over time $(F(1,920.15)=15.77, p<0.001)(\beta=$ $0.13, t=-4.184, p<0.001)$. There was no omnibus effect of Injury X Cue $(F(1,48.98)=0.97, p$ $=0.330)$. There was a significant omnibus effect of Injury X Time $(F(1,920.15)=34.72, p<$ 0.001) such that TBI rats decreased overall omissions over time while still omitting more than Sham rats $(\beta=-0.15, t=3.491, p<0.001)$ There was no omnibus Cue X Week effect $(F(1,920.15)=0.19, p=0.659)$. There was no omnibus effect of Injury X Time X Cue $(F(1,920.15)=1.22, p=0.269)$. See Figure 8 . 
Win-stay responses were examined using LMER [Fixed: Injury (Sham, TBI) x Cue (Cue, No Cue) x Time, Random: Subject]. There was no omnibus effect of Injury $(F(1,58.23)=0.00, p$ $=0.999)$. There was no omnibus effect of Cue $(F(1,58.23)=1.49, p=0.227)$. There was a significant omnibus effect of Time $(F(1,920.23)=495.67, p<0.001)$ such that win-stay responses increased over time $(\beta=0.15, t=5.872, p<0.001)$. There was a significant omnibus effect of Injury X Cue $(F(1,58.23)=5.37, p=0.024)$, however no significant differences were detected between TBI-Cue and Sham-Cue rats $(\beta=-0.40, t=-1.597, p=0.116)$, or TBI and Sham rats $(\beta=0.40, t=1.684, p=0.098)$. There was a significant omnibus effect of Injury $\mathrm{X}$ Time $(F(1,920.23)=9.04, p=0.003)$, however further analysis yielded no significant group differences $(\beta=0.03, t=0.932, p=0.351)$. There was a significant omnibus effect of Cue $\mathrm{X}$ Time $(F(1,920.23)=86.75, p<0.001)$, such that Cue rats had increased win-stay responses over time while rats without the cue remained relatively stable $(\beta=0.20, t=5.237, p<0.001)$. There was no omnibus effect of Injury X Cue X Time $(F(1,920.23)=2.99, p=0.084)$. See Figure 9. Lose-shift responses were examined using LMER [Fixed: Injury (Sham, TBI) x Cue (Cue, No Cue) x Time, Random: Subject]. There was no omnibus effect of Injury $(F(1,83.39)=$ $2.06, p=0.155)$. There was a significant omnibus effect of $\operatorname{Cue}(F(1,83.39)=17.55, p<0.001)$, such that Cue rats engaged in less lose-shift responses over time than No-Cue rats $(\beta=-0.67, t=$ $-2.483, p=0.015)$. There was a significant effect of Time $(F(1,920.46)=50.40, p<0.001)$ such but that effect washed out after further analysis $(\beta=0.002, t=0.051, p=0.960)$. There was no omnibus effect of Injury X Cue $(F(1,83.39)=0.35, p=0.555)$. There was no omnibus effect of Injury X Time $(F(1,920.46)=1.30, p=0.254)$. There was a significant omnibus effect of Cue $\mathrm{X}$ Time $(F(1,920.46)=103.19, p<0.001)$, such that Cue groups engaged in less lose-shift 
responses over time $(\beta=0.30, t=-5.759, p<0.001)$. There was no omnibus effect of Injury $\mathrm{X}$ Cue X Time $(F(1,920.46)=3.30, p=0.070)$. See Figure 10 .

\section{Lesion Analysis Experiments 1a \& $1 b$}

A one-way ANOVA was used to compare brain volumes between TBI, Sham, ShamCue, and TBI-Cue rats (Lesion Volume $\sim$ Group) yielded significant variation among groups $(F(3,36)=12.50, p<0.001)$. A Tukey post-hoc analysis was conducted $(p<0.05)$ : Sham and TBI were significantly different $(p=0.007)$; Sham-Cue and TBI-Cue were significantly different $(p<0.001)$; TBI and Sham-Cue were significantly different $(p=0.002)$; TBI-Cue and Sham were significantly different $(p<0.001)$. No other significant differences were detected. See Figure 11. There were no significant differences between original volume measurements $(\mathrm{M}=$ 11.33, $\mathrm{SD}=1.60)$ and IRA volume measurements $(\mathrm{M}=12.30, \mathrm{SD}=1.40) ;(t=0.91, p=0.398)$.

\section{Experiment 2: Differential Reinforcement of Low Rate Behavior}

Percent correct responses [correct / (incorrect + correct) $* 100]$ were analyzed using LMER [Fixed: Injury (Sham, TBI) x Cue (Cue, No Cue) x Time, Random: Subject]. There was no significant omnibus effect of Injury $(F(1,51.84)=1.03, p=0.314)$. There was a significant omnibus effect of Cue $(F(1,51.84)=155.58, p<0.001)$, such that Cue rats had greater percent correct responses than rats without the cue $(\beta=1.47, t=9.124, p<0.001)$. There was a significant omnibus effect of Time $(F(1,852.10)=577.00, p<0.001)$, such that percent correct responses increased over time $(\beta=0.25, t=14.526, p<0.001)$. There was no omnibus effect of Injury X Cue $(F(1,51.84)=0.001, p=0.937)$. There was a significant omnibus effect of Injury $\mathrm{X}$ Time $(F(1,852.10)=50.78, p<0.001)$, however that effect washed out after further analysis $(\beta=$ $0.03, t=1.439, p=0.151$. There was a significant omnibus effect of Cue $\mathrm{X}$ Time $(F(1,852.10)=$ 12.06, $p<0.001)$, however that effect washed out after further analysis $(\beta=0.03, t=1.176, p=$ 
0.240). There was a significant omnibus effect of Injury X Time X Cue $(F(1,852.10)=26.13, p$ $<0.001)$, such that no difference existed between TBI-Cue and Sham-Cue rats $(\beta=-0.03, t=-$ $1.439, p=0.151)$, but Sham rats had significantly increased percent correct responses over time compared to TBI rats $(\beta=-0.20, t=-8.579, p<0.001)$. These data are shown in Figure 12 .

IRT was analyzed using LMER [Fixed: Injury (Sham, TBI) x Cue (Cue, No Cue) x Time, Random: Subject]. There was no significant omnibus effect of Injury $(F(1,44.79)=1.10, p=$ 0.300). There was a significant omnibus effect of Cue $(F(1,44.79)=40.21, p<0.001)$, such that Cue rats had higher IRTs than No-Cue rats $(\beta=1.03, t=3.865, p<0.001)$. There was a significant omnibus effect of Time $(F(1,852.07)=541.21, p<0.001)$, such that IRTs increased over time $(\beta=0.37, t=17.336, p<0.001)$. There was no omnibus effect of Injury X Cue $(F(1,44.79)=1.05, p=0.311)$. There was a significant omnibus effect of Injury X Time $(F(1,852.07)=11.68, p<0.001)$, such that Sham rats increased IRTs over time compared to TBI rats $(\beta=0.15, t=4.710, p<0.001)$. There was a significant omnibus effect of Cue $\mathrm{X}$ Time $(F(1,852.07)=4.57, p=0.033)$, such that Cue rats increased IRTs over time compared to NoCue rats $(\beta=0.12, t=3.960, p<0.001)$. There was a significant omnibus effect of Injury $\mathrm{X}$ Time X Cue $(F(1,852.07)=10.95, p<0.001)$, such that no difference existed between TBI-Cue and Sham-Cue rats $(\beta=-0.00, t=-0.078, p=0.938)$, but Sham rats increased IRTs over time compared to TBI rats $(\beta=-0.15, t=-4.710, p<0.001)$. These data are shown on Figure 13 . Mean IRT for Sham was $14.74(\mathrm{SD}=3.94)$. Mean IRT for TBI was $10.76(\mathrm{SD}=3.94)$. Mean IRT for Sham-Cue was $18.63(\mathrm{SD}=3.67)$. Mean IRT for TBI-Cue was $18.99(\mathrm{SD}=5.84)$. Figure 14 is a histogram that displays the IRT distributions throughout the duration of the study. Total responses were analyzed using LMER [Fixed: Injury (Sham, TBI) x Cue (Cue, No Cue) x Time, Random: Subject]. There was no significant omnibus effect of Injury $(F(1,46.47)=$ 
$0.41, p=0.525)$. There was a significant omnibus effect of Cue $(F(1,46.47)=32.81, p<0.001)$, such that Cue rats had less total responses than No-Cue rats $(\beta=-1.07, t=3.698, p<0.001)$. There was a significant omnibus effect of Time $(F(1,852.08)=491.51, p<0.001)$, such that total responses decreased over time $(\beta=0.26, t=10.494, p<0.001)$. There was no omnibus effect of Injury X Cue $(F(1,46.47)=0.41, p=0.525)$. There was a significant omnibus effect of Injury $\mathrm{X}$ Time $(F(1,852.08)=10.57, p=0.001)$, however that effect washed out after further analysis $(\beta=$ $0.02, t=0.591, p=0.555$. There was no significant omnibus effect of Cue X Time $(F(1,852.08)$ $=0.0001, p=0.992)$. There was a significant omnibus effect of Injury X Time X Cue $(F(1,852.08)=63.67, p<0.001)$, such that no difference existed between TBI-Cue and ShamCue rats $(\beta=0.02, t=0.591, p=0.554)$, but Sham rats had significantly decreased responses over time compared to TBI rats $(\beta=-0.18, t=-5.113, p<0.001)$. These data are shown in Figure 15.

\section{Lesion Analysis Experiment 2}

A one-way ANOVA was used to compare brain volumes between TBI, Sham, ShamCue, and TBI-Cue rats (Lesion Volume $\sim$ Group) yielded significant variation among groups $(F(3,34)=28.06, p<0.001)$. A Tukey post-hoc analysis was conducted $(p<0.05)$ : Sham and TBI were significantly different $(p<0.001)$; Sham-Cue and TBI-Cue were significantly different $(p<0.001)$; TBI and Sham-Cue were significantly different $(p<0.001)$; TBI-Cue and Sham were significantly different $(p<0.001)$. No other significant differences were detected. See Figure 16. There were no significant differences between original volume measurements $(\mathrm{M}=$ $10.48, \mathrm{SD}=2.85)$ and IRA volume measurements $(\mathrm{M}=10.64, \mathrm{SD}=2.60) ;(t=0.08, p=0.936)$.

\section{DISCUSSION}

\section{Overview}


The current research demonstrates that injury-related cognitive deficits can be recovered by providing salient cues associated with correct responding. Deficits in behavioral flexibility and impulse control were both rescued to sham and near-sham levels respectively; this was done through a purely environmental manipulation. It is important to note that while impulse control and flexibility were considerably impaired, attention to visual stimuli remained largely intact; indicated by the improvements in performance associated with the increased saliency of environmental cues. The findings of these studies highlight a potential avenue for the development of effective behavioral therapeutics when considering TBI-related cognitive dysfunction. If post-injury rehabilitation models emphasize the identification and reinforcement of correct behavior while facilitating discrimination between desired and maladaptive behavior, TBI patients may be able to improve their quality of life substantially.

These studies showcase the therapeutic potential of the environment (in absence of any pharmaceutical intervention) to rescue cognitive deficits associated with severe frontal TBI. Post-injury therapeutics for TBI are currently underdeveloped, often resulting in enduring injuryrelated cognitive deficits (Maas et al., 2008) which has serious negative effects on quality of life for individuals suffering from this type of injury (CDC, 2019).

\section{Detection of Chronic Deficits}

Cognitive dysfunction associated with TBI can be quite profound in the acute post-injury phase (Brian, 1999). However, chronic cognitive deficits following TBI are often subtle in nature when compared to the former (Brian, 1999). In order to detect these deficits in preclinical research, it is necessary to use highly-sensitive behavioral tests. The current data suggest that chronic deficits in flexibility may be detectable by the PbR task, but not by the AST. Behavioral flexibility, in the form of reversals on the PbR task, was impaired following brain injury. In 
addition, TBI rats were severely impaired on DRL, our measure of impulse control. The robust behavioral deficits detected by $\mathrm{PbR}$ and $\mathrm{DRL}$ demonstrate the utility of repeated measures testing in operant chambers when examining chronic deficits in flexibility and impulse control.

AST and PbR both measure behavioral flexibility; AST did not detect differences in flexibility between Sham and TBI rats at 3 weeks post-injury in these experiments. There are several variables that may account for this lack of detection. First, PbR is a probabilistic task, meaning that the "correct" and "incorrect" responses are not truly correct and incorrect (which is why they were placed in quotations throughout the document) such that sometimes the "correct" response does not yield reinforcement, and sometimes the "incorrect" response does yield reinforcement. In contrast, AST is considered an "all-or-nothing" task. This means that correct responses always provide reinforcement and incorrect responses never provide reinforcement. The nature of an all or nothing task with two choices like the AST in these experiments is that that a rat can choose one side get $50 \%$ reinforcement. In contrast, the on $\mathrm{PbR}$, if a rat chooses one side it may only get $20 \%$ reinforcement. In addition, the probabilistic nature of $\mathrm{PbR}$ makes it a more difficult task, as the contingency is less detectable (less defined relationship between reinforcement and "correct" responding). The relative difficulty of each task is especially important when considering chronic deficits like the ones in this experiment. Previous research shows that chronic cognitive impairment following TBI is more subtle than acute impairment and may prove difficult to detect in rodents (Vonder Haar, Maas, Jacobs, \& Hoane, 2014). The probabilistic nature of $\mathrm{PbR}$ makes it a more sensitive task and capable of detecting these elusive chronic deficits. As well, PbR is a repeated measures task, whereas AST is a single measure task. This increases the resolution of $\mathrm{PbR}$ by facilitating a larger window to detect deficits. For example, visual inspection of Figure 8 shows that even for $\mathrm{PbR}$, no group differences were 
apparent until week 2-3. These data demonstrate the importance of choosing sensitive repeated measures tasks when examining chronic cognitive deficits in TBI.

Chronic deficits were also detected using the DRL task. This is suggestive of response perseveration or the inability to inhibit behavior that is no longer reinforced. Motivation to work for a reinforcer has been examined using the progressive ratio task (Vonder Haar, Maas, Jacobs, \& Hoane, 2014). This study found that rats with a frontal brain injury were less motivated to press a lever for a sugar pellet than control rats. A follow up study did not detect differences in breakpoint (number of times a rat will press a lever to receive a sugar pellet) between TBI and Sham rats, however TBI rats displayed inefficient response patterns typically associated with motivational deficits (Vonder Haar \& Winstanley, 2016). Generally, TBI rats continued pressing throughout the entire session, while Sham rats completed sessions early. When only considering the breakpoint variable, these data suggest that TBI rats are more motivated to work for sugar pellets than Sham rats; this is unlikely as clinical populations often suffer from major depressive disorder which is characterized by motivational deficits (Bombardier et al., 2010; Hershenberg et al., 2016). Due to the increased session duration in TBI rats, the progressive ratio data were instead interpreted as possible response perseveration, rather than greater motivation to respond (Vonder Haar \& Winstanley, 2016). Unpublished data from our lab comparing progressive ratio performance between sham and severe TBI rats replicated these results further supporting this hypothesis. The results of the current research support the response perseveration hypothesis, as performance on DRL was lower in TBI rats; TBI increased response perseveration on the DRL task resulting in less correct responses and more incorrect responses. It is important to note that because percent correct responses were used as the primary measurement of impulsivity, data on total responses was also included (Figure 15). 


\section{Cue Intervention}

It is difficult to determine if these deficits were truly due to impaired flexibility, or if the deficits are more associated with issues related to contingency detection/discrimination. Previous research found discrimination deficits were present when using a digging task that incorporates multiple senses (tactile, visual, olfactory) in frontally injured rats (Martens, Vonder Haar, Hutsell, \& Hoane, 2012). However, this research was conducted during the acute post injury phase and each specific discrimination was in effect for no more than 12 days. In addition, deficits were found in a simple tone discrimination task (Vonder Haar, Smith, French, Martens, Jacobs, \& Hoane, 2014). These deficits were also found primarily in the acute post injury phase ( $<25$ days). A major difference between the current research and the previously discussed research is that the testing phase was greater than 25 days post injury (to examine chronic deficits). AST performance in the current research, in which TBI rats demonstrated no deficits despite the large sample size, suggests that the general ability to discriminate was largely intact during the chronic post-injury phase. This is further supported by substantial increases in performance associated with the cue intervention in $\mathrm{PbR}$.

Clinical TBI is often characterized by impulsive aggression (Wood \& Thomas, 2013) and impulsive sexual behavior (Moreno \& McKerral, 2018); we interpret the poor performance of TBI rats on DRL as the preclinical manifestation of TBI-induced impulsivity or an inability to inhibit responding when the contingency requires it. In addition, previous research comparing sham and TBI performance on a Peak Interval schedule (that required precisely timed responses to obtain reinforcement) demonstrated that timing behavior may be impaired in TBI rats and may contribute to response inhibition (Scott \& Vonder Haar, 2018). This may have contributed to poor performance on DRL as well, as it is a time-based schedule. Visual examination of the DRL 
IRT distributions displayed in Figure 14 suggest that deficits in DRL performance may be associated with a combination of impulse control, timing, and attention. However, a more indepth analysis of these data must be conducted in order to determine any definitive conclusions. Generally speaking, TBI rat IRT distributions are lower than that of Sham rats, which may be indicative of response inhibition. The addition of cues caused both the distributions of the Sham and TBI rats to both move closer to the optimal IRT ( $20 \mathrm{~s})$, however TBI data were more spread of than Sham data. This may implicate issues with timing in combination with attention. While TBI rats did not recover to Sham level performance on DRL, the TBI rat's ability to attend to visual cues during the chronic post injury phase remained largely intact, as the performance was greatly improved by cues, almost reaching Sham levels. However, the compound effects of poor timing, impaired impulse control, and attentional deficits may explain why the cue treatment improved TBI performance substantially, but not to Sham levels, as we saw in PbR.

\section{Injury Model}

Flexibility and impulse control are both frontally-mediated behaviors; the injury model chosen for this experiment destroys the majority of the medial PFC. It is important to consider why it is so difficult to detect chronic deficits following such a severe injury. Following a brain injury, compensatory changes to structure and function of the brain, called neuroplastic changes, may occur (Chen, Epstein, \& Sterm, 2010). Therefore, in addition to the test-related variables and various cognitive deficits, performance may also be affected by injury-induced neuroplastic changes. While flexibility is generally associated with the PFC and OFC (Butts et al., 2013), impulse control is more specifically associated with the PFC (Sokolowski \& Salamone, 1994). The OFC is largely intact in this model and that may explain why TBI rats improved to Sham levels in PbR but not DRL with the cue intervention. In addition, simple stimulus-response 
relationships are generally associated with the OFC (Schoenbaum \& Roesch, 2005). It is possible that due to the lack of PFC, the behavior of the TBI rats in these experiments was primarily mediated by the OFC, so while the TBI rats had generally poor performance, the addition of the cues facilitated the development of a simple stimulus-response relationship that improved performance. This should be explored further using immunohistochemistry to examine activity in the brain areas associated with these behaviors.

It is possible that injury model used in these studies affected the results. The CCI model was chosen due to its precision and the focal injury it produces. The current research was specifically interested in examining deficits associated with severe-frontal injury, however in clinical populations, TBI is not often isolated to a single brain area. It would be useful to reexamine these deficits using different injury models to improve the translational validity. In particular it would be useful to determine if these deficits still exist when using a non-penetrative model such as the CHIMERA or blast overpressure model. It would also be useful to reexamine outcomes when using an open head injury model that causes more distal damage, like the FPI.

\section{Therapeutic Implications}

While significant deficits were detected between Sham and TBI rats on PbR and DRL, the cue intervention improved TBI performance to Sham and near Sham levels, respectively. These results highlight the potential therapeutic effects of environmental manipulation through increasing the saliency of cues associated with correct responding. Previous research that has detected significant deficits in cue detection in the acute phases (Martens, Vonder Haar, Hutsell, \& Hoane, 2012; Vonder Haar, Smith, French, Martens, Jacobs, \& Hoane, 2014). The current research suggests that TBI causes chronic impairment to PbR and DRL performance, however, the severity of those impairments appears to be less than what is generally seen in the acute post- 
injury phase. While the severity of chronic deficits is usually less than what is seen during the acute phase, the subtle environmental cues associated with each contingency (e.g., reinforcement, time) were not enough to facilitate performance at the level of Sham rats. The cue manipulation provided salient cues associated with correct responding that were detectable by the injured rats. This suggests that while cue-based rehabilitative strategies may be effective for treating cognitive dysfunction during the chronic post injury phases, clinical populations may not benefit from them during the acute post injury phase. It may be advantageous to initially prioritize the treatment of sensorimotor dysfunction associated with the injury in the acute phase, as stroke (similar pathophysiology to TBI) research suggests there may be an early window of opportunity for improving motor deficits (Biernaskie, Chernenko, \& Corbett, 2004); this should be followed by patient-specific cognitive skills training. In addition, it is important to be aware that there may be compounded effects of impaired attention, impulse control, and timing; these data suggest impulsive behavior may be more difficult to treat than deficits in flexibility, which were recovered to Sham levels with the cue manipulation in this study.

Medical practitioners often use pharmaceutical interventions for behavioral deficits in non-TBI populations (Locher et al., 2017), however treatments have not translated well into TBI populations and have not resulted in any successful clinical trials (Silverberg et al., 2017). The current research may inform the further development of therapeutic strategies for behavioral deficits associated with TBI. The development of new behavioral therapeutics may prove efficacious for improving outcomes further; these should emphasize the identification and reinforcement of appropriate or "correct" behaviors.

While the current research suggests that behavioral therapeutics may sufficient for treating chronic deficits, the development of effective pharmacotherapeutics may facilitate 
further improvement. For example, amphetamines have demonstrated promising results when used to treat cognitive deficits in preclinical research (Vonder Haar et al, 2016). In addition, combination therapy (pharmaceutical + behavioral), is highly effective in treating certain disorders. For example, selective serotonin reuptake inhibitors (SSRIs) by themselves did not affect peak cortisol levels in adults with generalized anxiety disorder, unless they were used in combination with cognitive behavioral therapy (Rosnick et al., 2016). The effort to establish pharmaceutical interventions for TBI may be augmented if effective behavioral therapeutics exist, such that the combination effect of the two therapeutic approaches will yield significant increases in behavioral outcomes, where solely pharmaceutical interventions have previously failed.

\section{Future Directions}

The current research identifies several potential avenues for future research. In particular, future research should examine behavioral therapeutics in combination with pharmaceuticals; this may augment performance even further. Amphetamines increase the activity of dopamine, norepinephrine, and serotonin in the brain (Kuczenski \& Segal, 1997), so other drugs that improve the function of these neurotransmitters are logical targets for future research. This approach may facilitate the recovery of DRL deficits in TBI rats to Sham levels. In addition, it may be advantageous to conduct a more advanced histological measurement to identify discrepancies in neurotransmitter activity in the associated brain areas between cued and noncued rats; this may help to identify potential targets for pharmacological intervention. In particular, the examination of neurotransmitter activity in the brain areas associated with flexibility, impulse control, and attention may yield interesting results. 
It would also be useful to reexamine these deficits using different injury models to improve translational validity. In particular, it would be useful to determine if these deficits still occur when using a non-penetrative model such as the CHIMERA or blast overpressure model. As well, a reexamination of outcomes when using other open head models would provide further insights. For example, an open head injury model that causes more distal damage, like the FPI, may differentially affect behavioral outcomes when compared to the highly-focal CCI model that was used in the current research.

Adjusting the parameters associated with each respective behavioral test may provide useful insights on the nature of these deficits. Researchers should attempt to determine the influence of probability on $\mathrm{PbR}$ performance. This could be done by using $\mathrm{PbR}$ with assured outcomes (Dalton et al., 2014), or examining the probabilities associated with "correct" and “incorrect" responding parametrically (e.g., 100\%-0\% vs. 80\%-20\% vs. 60\%-40\% vs. 50\%50\%). AST could also be reexamined at different post-injury time points to determine when cognition recovers to a level that is treatable using our cue manipulation. It may also be useful to examine deficits in impulse control using a more complex behavioral test such as the stop signal response task (Verbruggen et al., 2019). The complexity of this task may better replicate the intricacies of the human experience, facilitating better translation into human populations. As well modifications to the cue treatment could be examined. Some potential approaches would be to examine different types of stimuli (e.g. tone vs. light), or a parametric analyses of stimulus saliency and the associated treatment efficacy.

In a clinical setting, it may be beneficial to consider these data when evaluating effective behavioral therapeutics for TBI patients. Emphasis on clearly determining problem behavior and 
providing appropriate alternatives may improve outcomes associated with behavioral intervention. 


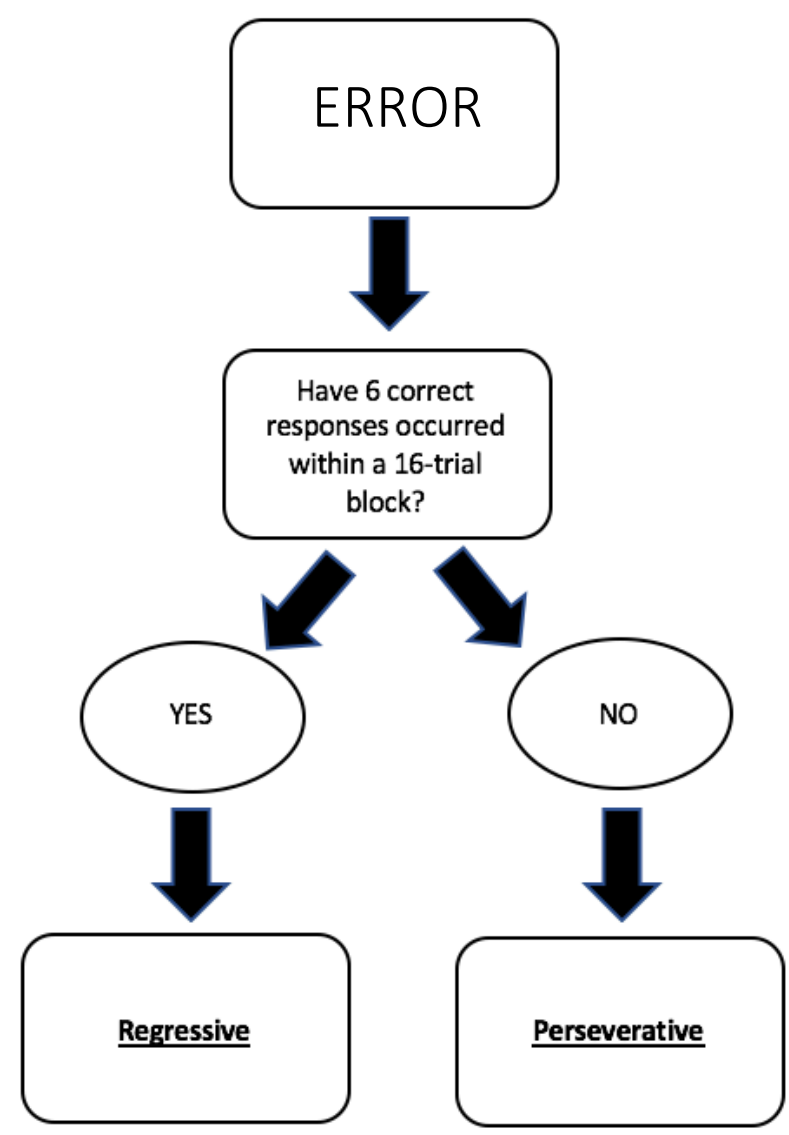

Figure 1. Flow chart of qualitative error analysis for Phase 3 (reversal) of Experiment 1a. 


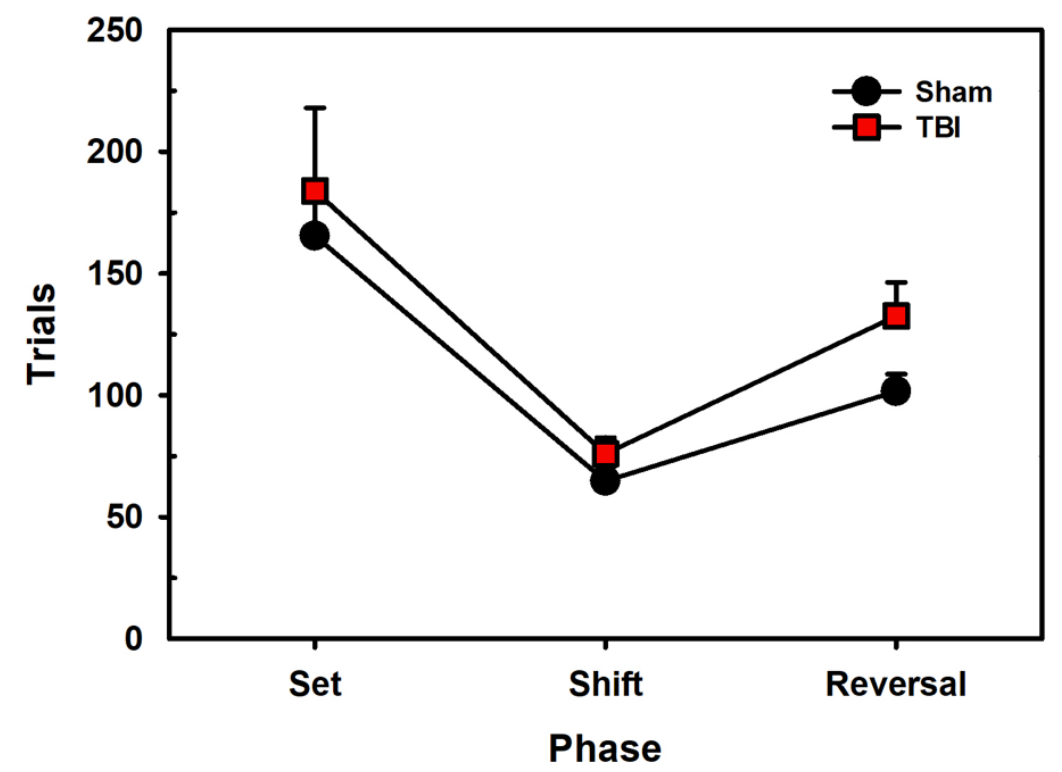

Figure 2. Trials to criterion for AST were analyzed using a 2x3 repeated measures ANOVAs [Injury (Sham, TBI) X Phase (P1, P2, P3)]. There was no Injury X Phase interaction $(F(1,117)=$ $0.274, p=0.761)$. 


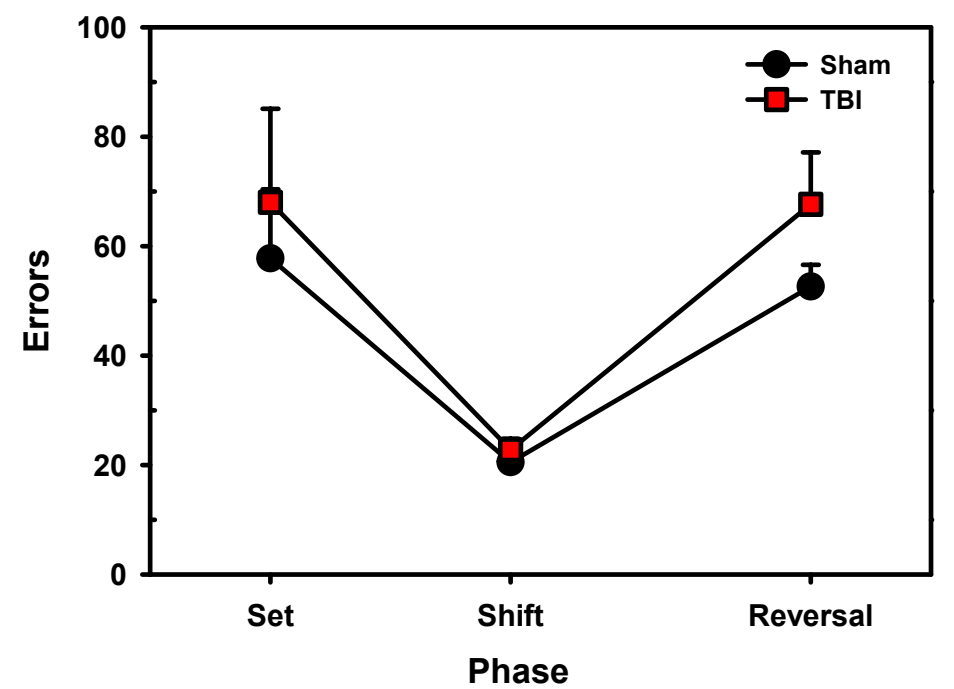

Figure 3. Errors to criterion for AST were analyzed using a 2x3 repeated measures ANOVAs [Injury (Sham, TBI) X Phase (P1, P2, P3)]. There was no Injury X Phase interaction $(F(1,117)=$ $0.153, p=0.850)$. 


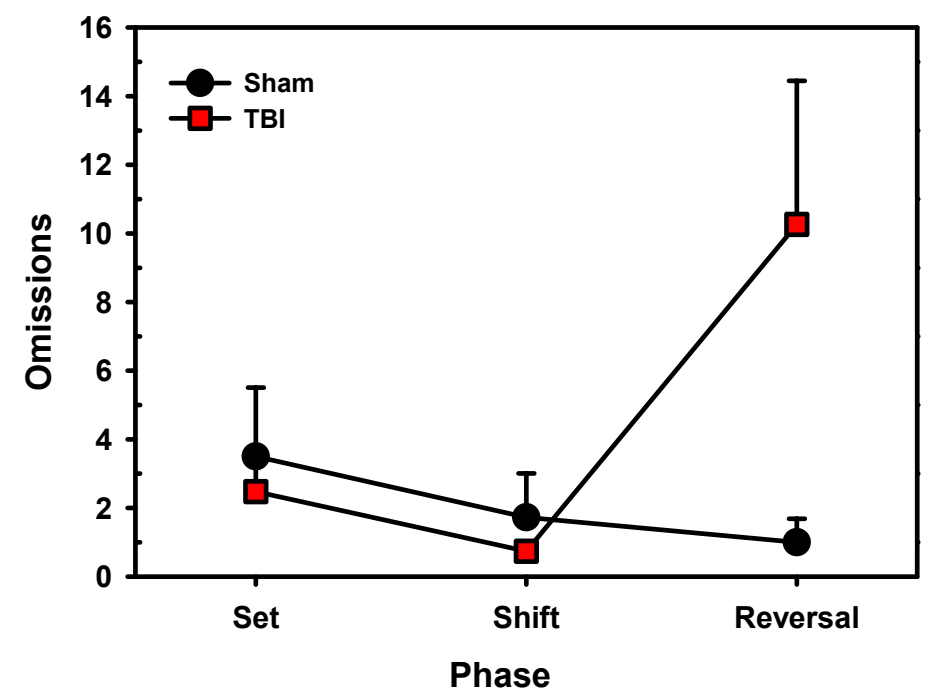

Figure 4. Omissions for AST were analyzed using a 2x3 repeated measures ANOVAs [Injury (Sham, TBI) X Phase (P1, P2, P3)]. There was a significant Injury X Phase interaction for omissions $(F(1,117)=4.185, p=0.013)$. Post-hoc analyses using 2-sample T-Tests revealed that TBI rats had significantly more omissions than Sham rats in Phase $3(t(18.96)=-2.18, p=$ 0.042). 


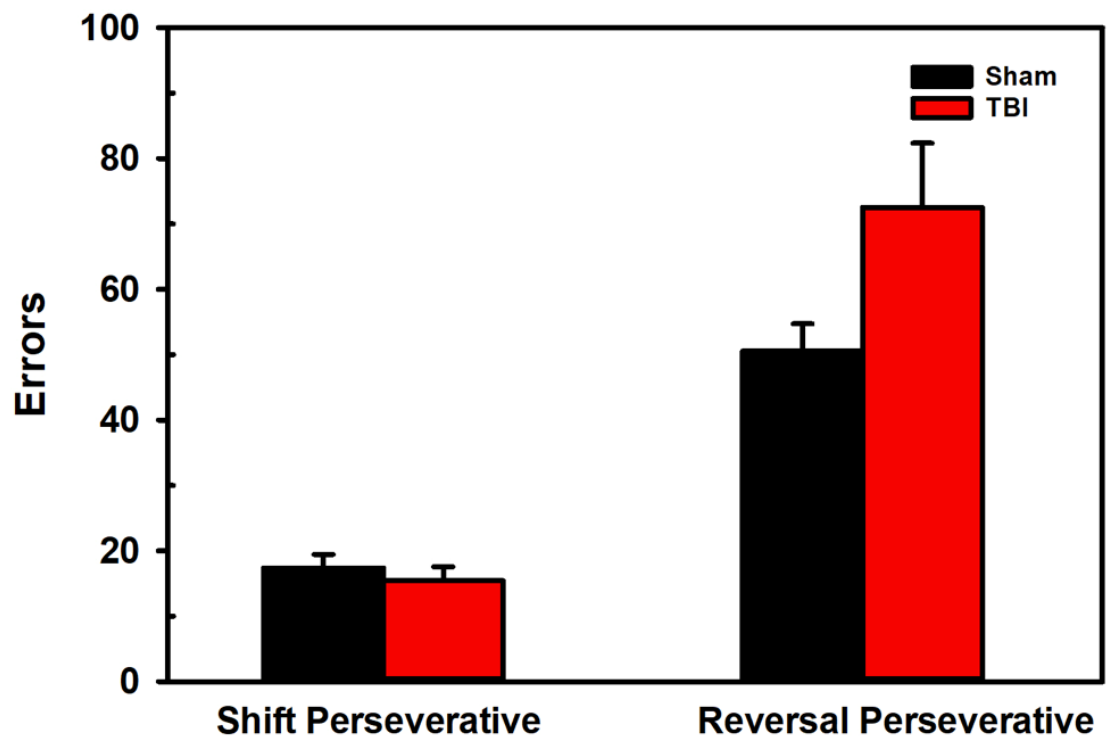

Figure 5. A $2 \times 2$ repeated measures ANOVA [Injury (Sham, TBI) $x$ Phase (P2, P3)] was used to examine perseverative errors in AST. There were no significant between Injury X Phase interactions for perseverative errors $(F(1,78)=3.830, p=0.054)$. 


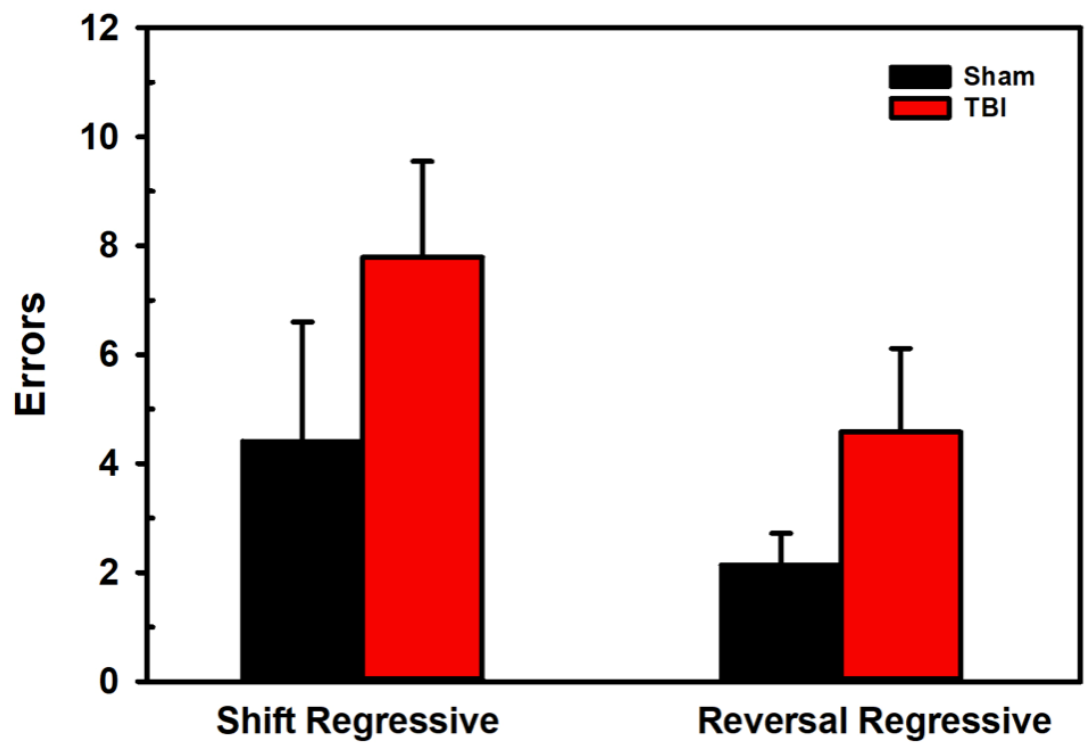

Figure 6. A $2 \times 2$ repeated measures ANOVA [Injury (Sham, TBI) x Phase (P2, P3)] was used to examine regressive errors in AST. There were no significant between Injury X Phase interactions for regressive errors $(F(1,78)=0.809, p=0.371)$. 


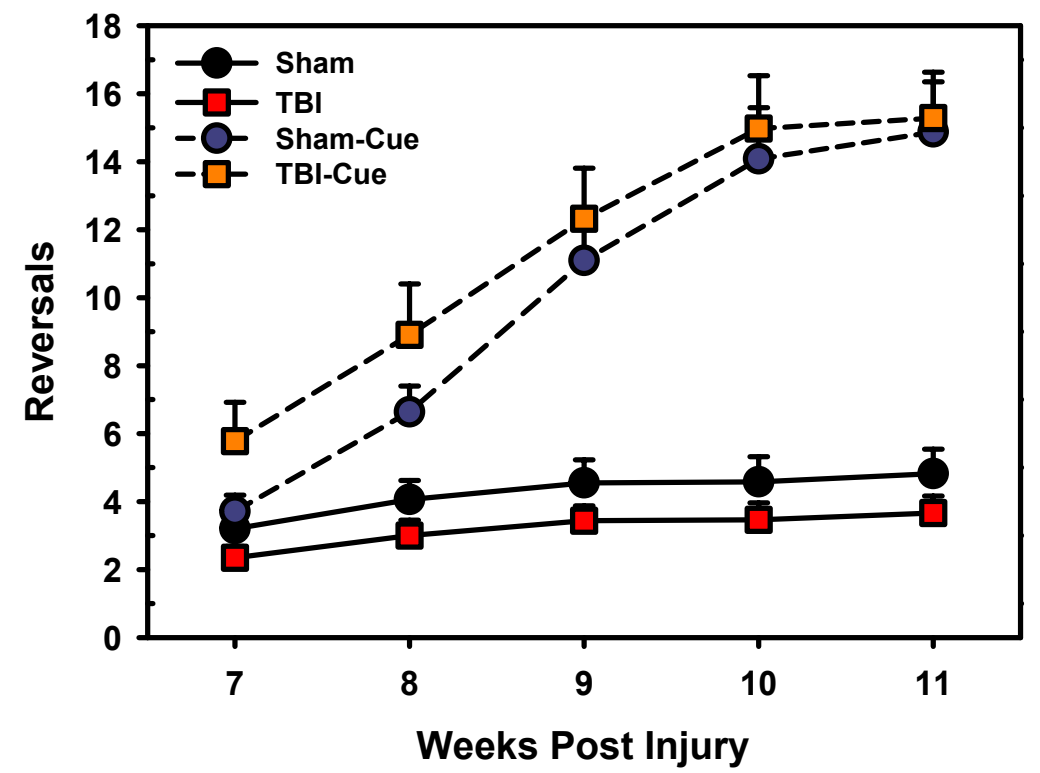

Figure 7. Reversals for trials completed (accounting for omissions) on $\mathrm{PbR}$. There was a significant omnibus effect of Injury X Cue $(F(1,76.02)=9.83, p=0.002)$ such that the TBI-Cue group achieved more reversals than the Sham-Cue group $(\beta=0.36, t=-2.022, p=0.046)$, and the Sham group achieved more reversals than the TBI group $(\beta=0.47, t=-2.423, p=0.018)$. 


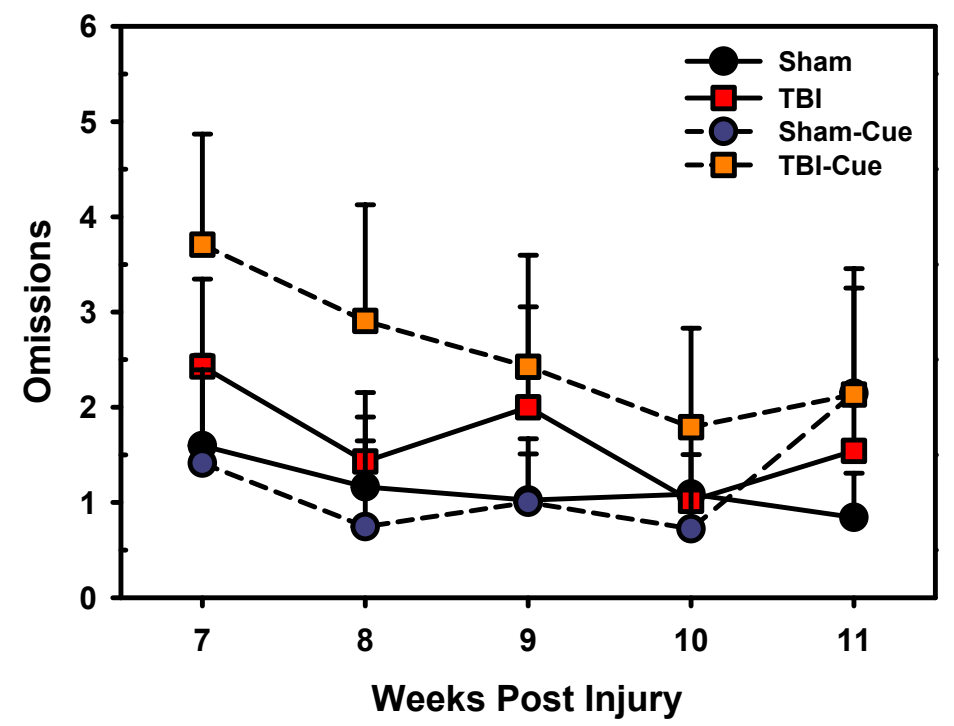

Figure 8. Omissions on PbR were examined using LMER [Fixed: Injury (Sham, TBI) x Cue (Cue, No Cue) x Time, Random: Subject]. There was a significant omnibus effect of Injury such that TBI rats omitted more than Sham rats overall $(F(1,48.98)=13.69, p<0.001)$. 


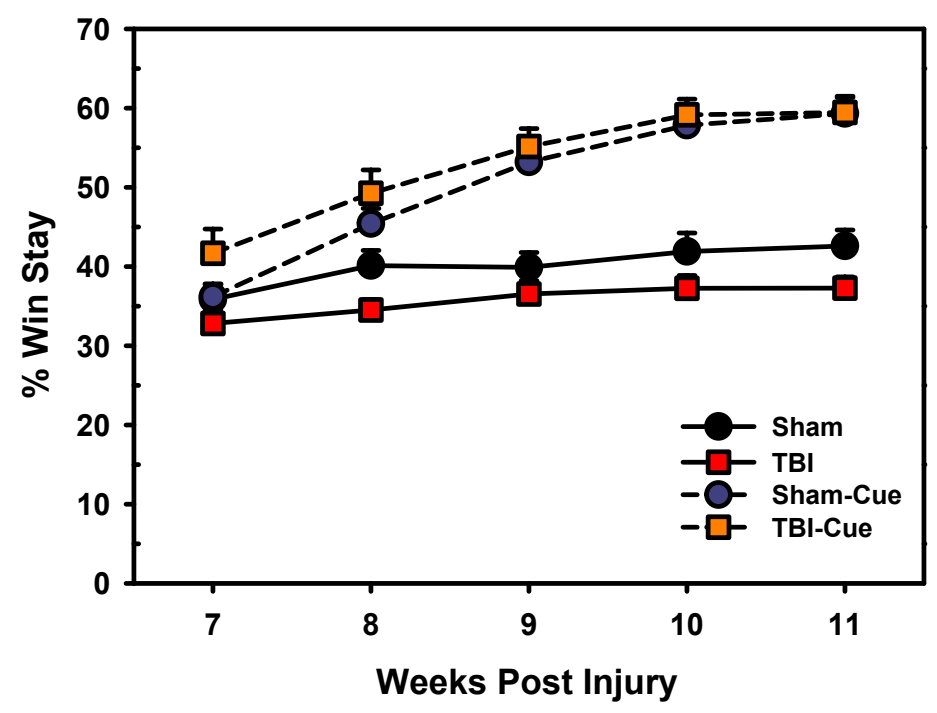

Figure 9. Win-stay responses were examined using LMER [Fixed: Injury (Sham, TBI) x Cue (Cue, No Cue) x Time, Random: Subject]. There was a significant omnibus effect of Cue X Time $(F(1,920.23)=86.75, p<0.001)$, such that Cue rats had increased win-stay responses over time while No Cue rats remained relatively stable. 


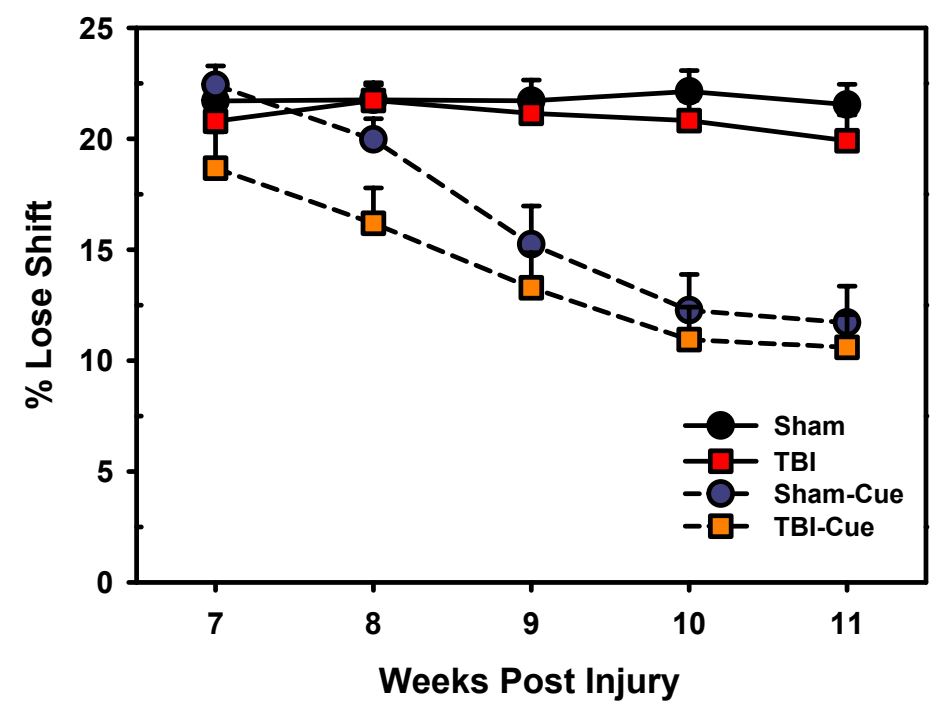

Figure 10. Lose-shift responses were examined using LMER [Fixed: Injury (Sham, TBI) x Cue (Cue, No Cue) x Time, Random: Subject]. There was a significant effect of Time $(F(1,920.46)=$ $50.40, p<0.001)$ such that lose-shift responses decreased over time. 


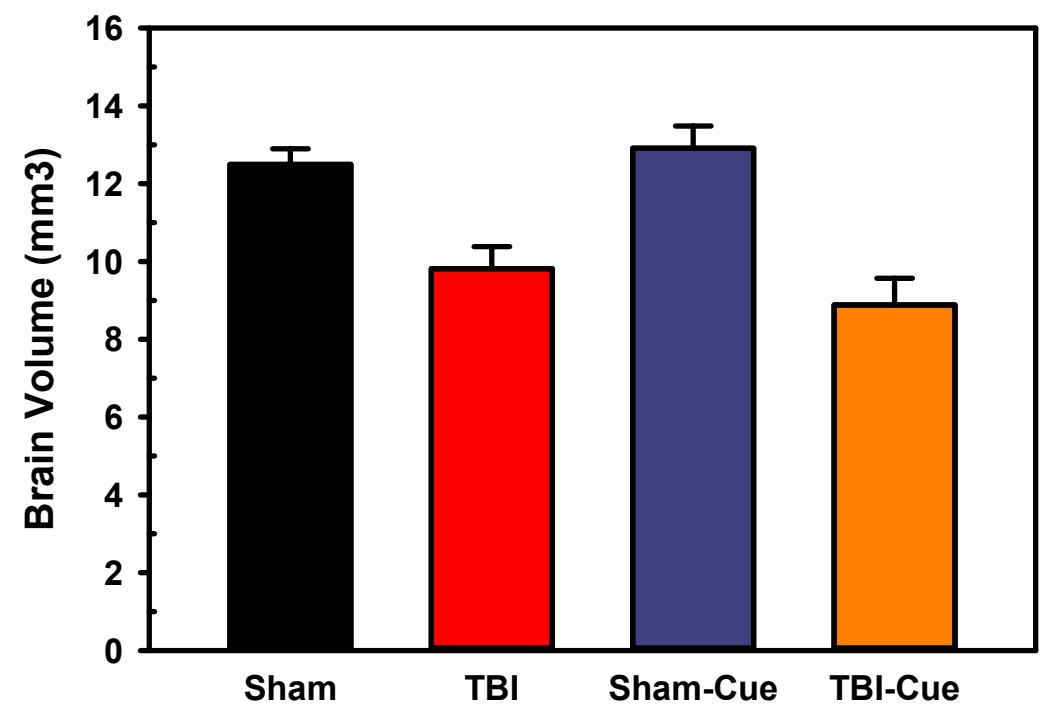

Figure 11. A one-way ANOVA was used to compare brain volumes between TBI, Sham, ShamCue, and TBI-Cue rats (Lesion Volume $\sim$ Group) yielded significant variation among groups $(F(3,36)=12.50, p<0.001)$. A Tukey post-hoc analysis was conducted $(p<0.05)$ : Sham and TBI were significantly different $(p=0.007)$; Sham-Cue and TBI-Cue were significantly different $(p<0.001)$; TBI and Sham-Cue were significantly different $(p=0.002)$; TBI-Cue and Sham were significantly different $(p<0.001)$. No other significant differences were detected. 


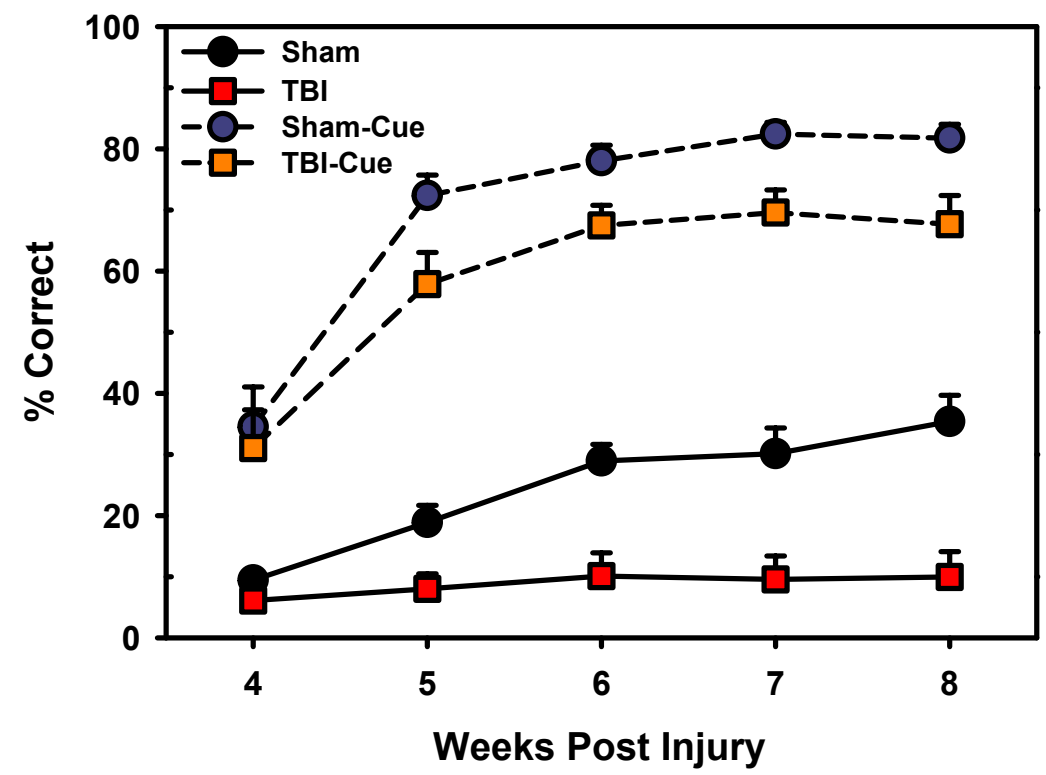

Figure 12. Percent correct responses [correct / (incorrect + correct) * 100] were analyzed using LMER [Fixed: Injury (Sham, TBI) x Cue (Cue, No Cue) x Time, Random: Subject].

There was a significant omnibus effect of Injury X Time X Cue $(F(1,852.10)=26.13, p<$ 0.001), such that no difference existed between TBI-Cue and Sham-Cue rats $(\beta=-0.03, t=-$ $1.439, p=0.151)$, but Sham rats had significantly increased percent correct responses over time compared to TBI rats $(\beta=-0.20, t=-8.579, p<0.001)$. 


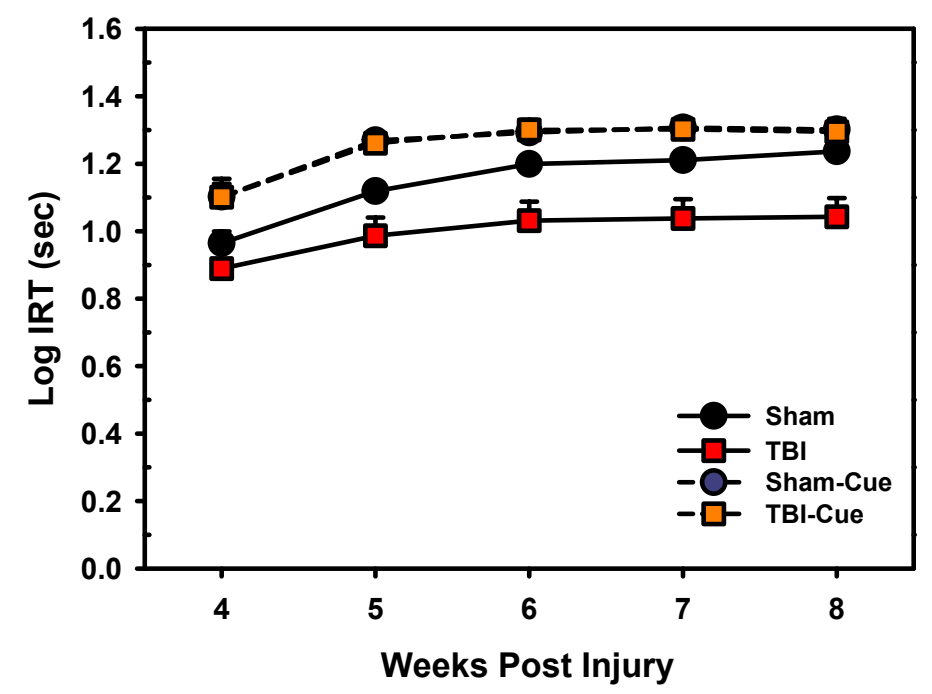

Figure 13. IRT was analyzed using LMER [Fixed: Injury (Sham, TBI) x Cue (Cue, No Cue) x Time, Random: Subject]. There was a significant omnibus effect of Injury X Time X Cue $(F(1,852.07)=10.95, p<0.001)$, such that no difference existed between TBI-Cue and ShamCue rats $(\beta=-0.00, t=-0.078, p=0.938)$, but Sham rats increased IRTs over time compared to TBI rats $(\beta=-0.15, t=-4.710, p<0.001)$. 


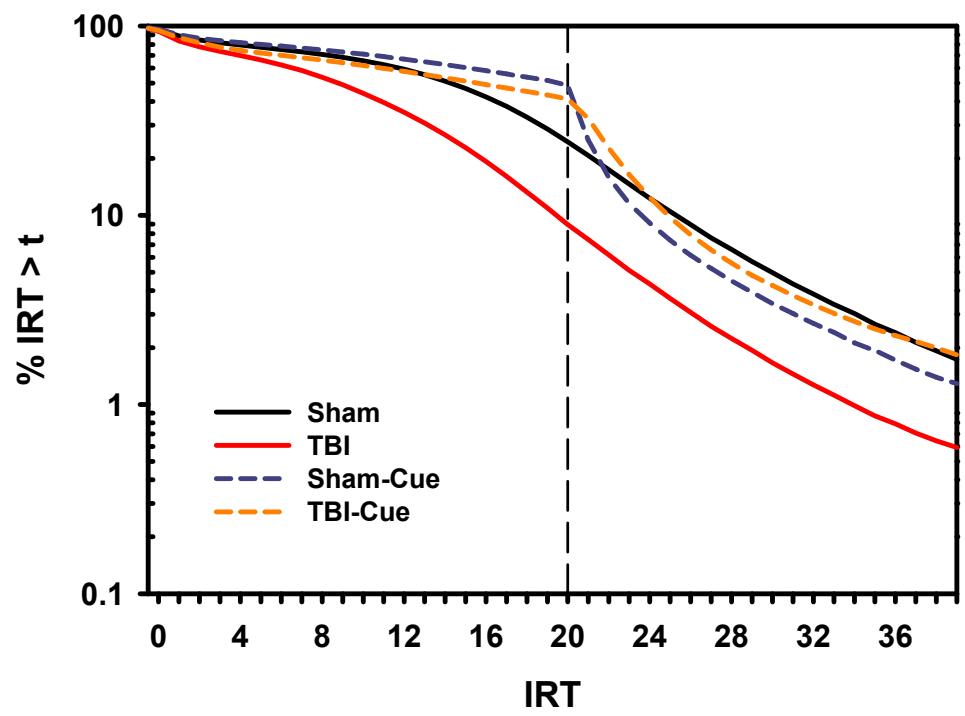

Figure 14. Line graph of cumulative $\log$ IRT distributions $(\log \%$ IRT $>$ ) for all DRL sessions. Log \% IRT is represented on the $\mathrm{y}$-axis and IRT is represented on the x-axis. A verticle black dotted line represents the optimal response (DRL 20). 


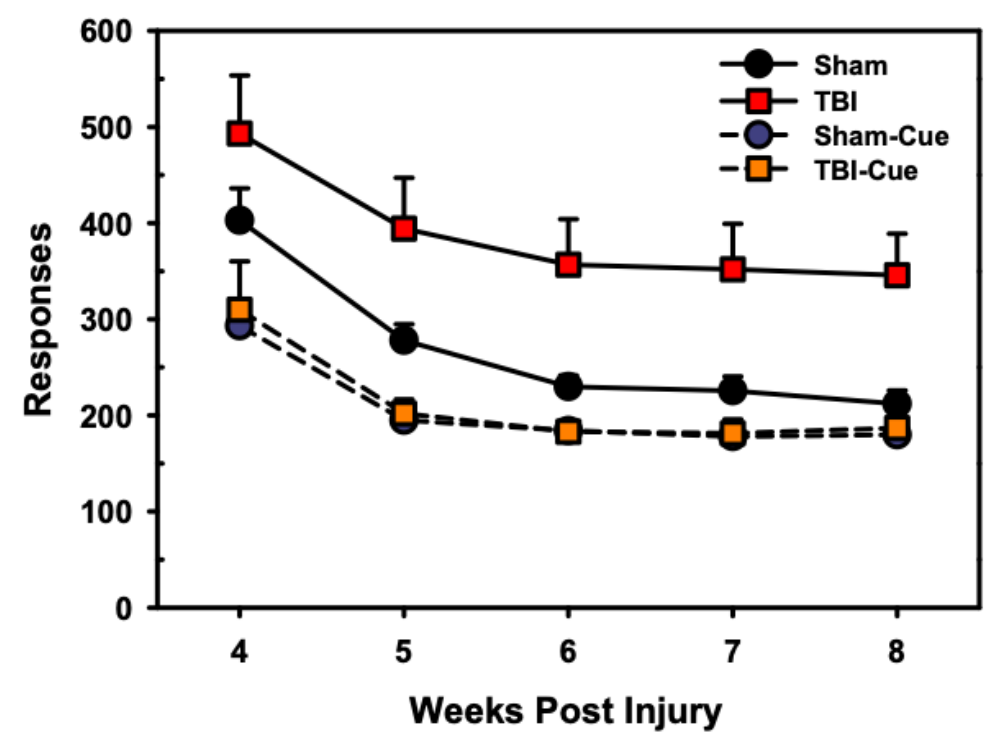

Figure 15. Total Response was analyzed using LMER [Fixed: Injury (Sham, TBI) x Cue (Cue, No Cue) x Time, Random: Subject]. There was a significant omnibus effect of Injury X Time X Cue $(F(1,852.08)=63.67, p<0.001)$, such that no difference existed between TBI-Cue and Sham-Cue rats $(\beta=0.02, t=0.591, p=0.554)$, but Sham rats had significantly decreased responses over time compared to TBI rats $(\beta=-0.18, t=-5.113, p<0.001)$. 


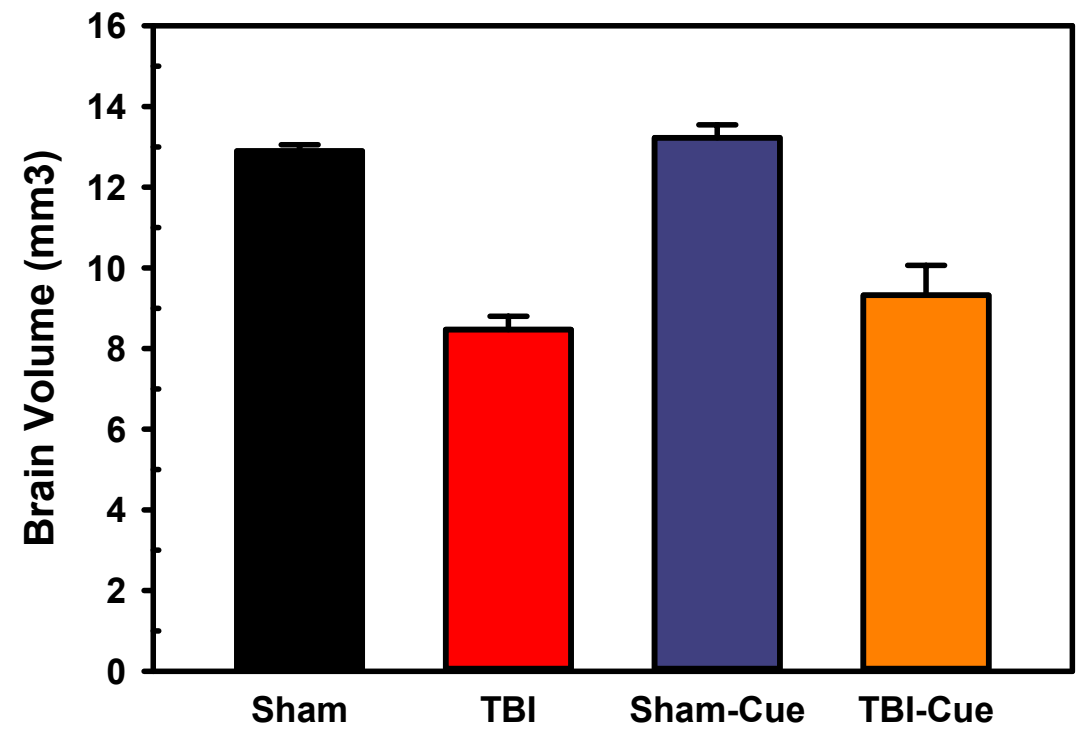

Figure 16. A one-way ANOVA was used to compare brain volumes between TBI, Sham, ShamCue, and TBI-Cue rats (Lesion Volume $\sim$ Group) yielded significant variation among groups $(F(3,34)=28.06, p<0.001)$. A Tukey post-hoc analysis was conducted $(p<0.05)$ : Sham and TBI were significantly different $(p<0.001)$; Sham-Cue and TBI-Cue were significantly different $(p<0.001)$; TBI and Sham-Cue were significantly different $(p<0.001)$; TBI-Cue and Sham were significantly different $(p<0.001)$. No other significant differences were detected. 


\section{References}

Ahlers, S. T., Vasserman-Stokes, E., Shaughness, M. C., Hall, A. A., Shear, D. A., Chavko, M., ... \& Stone, J. R. (2012). Assessment of the effects of acute and repeated exposure to blast overpressure in rodents: toward a greater understanding of blast and the potential ramifications for injury in humans exposed to blast. Frontiers in neurology, 3, 32.

Amodeo, L. R., McMurray, M. S., \& Roitman, J. D. (2017). Orbitofrontal cortex reflects changes in response--outcome contingencies during probabilistic reversal learning. Neuroscience, 345, 27-37.

Braun, C. M., Daigneault, S., \& Champagne, D. (1989). Information processing deficits as indexed by reaction time parameters in severe closed head injury. International Journal of Clinical Neuropsychology, 11(4), 167-176.

Bayly, P. V., Cohen, T. S., Leister, E. P., Ajo, D., Leuthardt, E. C., \& Genin, G. M. (2005). Deformation of the human brain induced by mild acceleration. Journal of neurotrauma, 22(8), 845-856.

Biernaskie, J., Chernenko, G., \& Corbett, D. (2004). Efficacy of rehabilitative experience declines with time after focal ischemic brain injury. Journal of Neuroscience, 24(5), 1245-1254.

Bombardier, C. H., Fann, J. R., Temkin, N. R., Esselman, P. C., Barber, J., \& Dikmen, S. S. (2010). Rates of major depressive disorder and clinical outcomes following traumatic brain injury. Jama, 303(19), 1938-1945.

Bondi, C. O., Semple, B. D., Noble-Haeusslein, L. J., Osier, N. D., Carlson, S. W., Dixon, C. E., ... \& Kline, A. E. (2015). Found in translation: Understanding the biology and behavior 
of experimental traumatic brain injury. Neuroscience \& Biobehavioral Reviews, 58, 123 146.

Brady, A. M., \& Floresco, S. B. (2015). Operant procedures for assessing behavioral flexibility in rats. Journal of visualized experiments: JoVE, (96).

Brian, T. (1999). Rehabilitation of persons with traumatic brain injury. JAMA, 282(10), 974-983.

Brown, P. L., \& Jenkins, H. M. (1968). Auto-shaping of the pigeon's key-peck. Journal of the experimental analysis of behavior, 11(1), 1-8.

Butts, K. A., Floresco, S. B., \& Phillips, A. G. (2013). Acute stress impairs set-shifting but not reversal learning. Behavioural brain research, 252, 222-229.

Center for Disease Control. (2019). Traumatic brain injury \& concussion. Retrieved August 23, 2019.

Cernak, I., \& Noble-Haeusslein, L. J. (2010). Traumatic brain injury: an overview of pathobiology with emphasis on military populations. Journal of Cerebral Blood Flow \& Metabolism, 30(2), 255-266.

Chen, H., Epstein, J., \& Stern, E. (2010). Neural plasticity after acquired brain injury: evidence from functional neuroimaging. $P M \& R, 2, \mathrm{~S} 306-\mathrm{S} 312$.

Coggeshall, R. E. (1992). A consideration of neural counting methods. Trends in Neurosciences, 15(1), 9-13.

Cole, J.T., Yarnell, A., Kean, W.S., Gold, E., Lewis, B., Ren, M., McMullen, D.C., Jacobowitz, D.M., Pollard, H.B., O'Neill, J.T. and Grunberg, N.E. (2011). Craniotomy: True sham for traumatic brain injury, or a sham of a sham? Journal of Neurotrauma, 28, 359-369. 
Costa, V. C. I., Bueno, J. L. O., \& Xavier, G. F. (2005). Dentate gyrus-selective colchicine lesion and performance in temporal and spatial tasks. Behavioural brain research, 160(2), 286303.

Cox, B. M., Cope, Z. A., Parsegian, A., Floresco, S. B., Aston-Jones, G., \& See, R. E. (2016). Chronic methamphetamine self-administration alters cognitive flexibility in male rats. Psychopharmacology, 233(12), 2319-2327.

Curtis, S. D., \& Nonneman, A. J. (1977). Effects of successive bilateral hippocampectomy on DRL 20 performance in rats. Physiology \& behavior, 19(6), 707-712.

Dalton, G. L., Phillips, A. G., \& Floresco, S. B. (2014). Preferential involvement by nucleus accumbens shell in mediating probabilistic learning and reversal shifts. Journal of Neuroscience, 34(13), 4618-4626.

Dalton, G. L., Wang, N. Y., Phillips, A. G., \& Floresco, S. B. (2016). Multifaceted contributions by different regions of the orbitofrontal and medial prefrontal cortex to probabilistic reversal learning. Journal of Neuroscience, 36(6), 1996-2006.

Diaz, R., Robbins, T. W., \& Roberts, A. C. (1996). Dissociation in prefrontal cortex of affective and attentional shifts. Nature, 380(6569), 69-72.

Diergaarde, L., Pattij, T., Poortvliet, I., Hogenboom, F., de Vries, W., Schoffelmeer, A. N., \& De Vries, T. J. (2008). Impulsive choice and impulsive action predict vulnerability to distinct stages of nicotine seeking in rats. Biological psychiatry, 63(3), 301-308.

Ellen, P., \& Butter, J. (1969). External cue control of DRL performance in rats with septal lesions. Physiology \& Behavior, 4(1), 1-6. 
Farina, F. R., Burke, T., Coyle, D., Jeter, K., McGee, M., O’Connell, J., ... \& Commins, S. (2015). Learning efficiency: the influence of cue salience during spatial navigation. Behavioural processes, 116, 17-27.

Faul, M., \& Coronado, V. (2015). Epidemiology of traumatic brain injury. In Handbook of clinical neurology (Vol. 127, pp. 3-13). Elsevier.

Feeney, D. M., Boyeson, M. G., Linn, R. T., Murray, H. M., \& Dail, W. G. (1981). Responses to cortical injury: I. Methodology and local effects of contusions in the rat. Brain research, $211(1), 67-77$.

Ferster, C. B., \& Skinner, B. F. (1957). Schedules of Reinforcement. East Norwalk, CT: Appleton-Century-Crofts.

Green, R. E., Turner, G. R., \& Thompson, W. F. (2004). Deficits in facial emotion perception in adults with recent traumatic brain injury. Neuropsychologia, 42(2), 133-141.

Hershenberg, R., Satterthwaite, T. D., Daldal, A., Katchmar, N., Moore, T. M., Kable, J. W., \& Wolf, D. H. (2016). Diminished effort on a progressive ratio task in both unipolar and bipolar depression. Journal of affective disorders, 196, 97-100.

Hill, D. F., Parent, K. L., Atcherley, C. W., Cowen, S. L., \& Heien, M. L. (2018). Differential release of dopamine in the nucleus accumbens evoked by low-versus high-frequency medial prefrontal cortex stimulation. Brain stimulation, 11(2), 426-434.

Hoane, M. R., Akstulewicz, S. L., \& Toppen, J. (2003). Treatment with vitamin B 3 improves functional recovery and reduces GFAP expression following traumatic brain injury in rats. Journal of Neurotrauma, 20(11), 1189-1199. 
Hoofien, D., Gilboa, A., Vakil, E., \& Donovick, P. J. (2001). Traumatic brain injury (TBI) 10 ? 20 years later: a comprehensive outcome study of psychiatric symptomatology, cognitive abilities and psychosocial functioning. Brain injury, 15(3), 189-209.

Junn, C., Bell, K. R., Shenouda, C., \& Hoffman, J. M. (2015). Symptoms of concussion and comorbid disorders. Current pain and headache reports, 19(9), 46.

Kim, J., Wasserman, E. A., Castro, L., \& Freeman, J. H. (2016). Anterior cingulate cortex inactivation impairs rodent visual selective attention and prospective memory. Behavioral neuroscience, $130(1), 75$.

Kocka, A., \& Gagnon, J. (2014). Definition of impulsivity and related terms following traumatic brain injury: a review of the different concepts and measures used to assess impulsivity, disinhibition and other related concepts. Behavioral Sciences, 4(4), 352-370.

Kuczenski, R., \& Segal, D. S. (1997). Effects of methylphenidate on extracellular dopamine, serotonin, and norepinephrine: comparison with amphetamine. Journal of neurochemistry, 68(5), 2032-2037.

Levin, H. S., Culhane, K. A., Mendelsohn, D., Lilly, M. A., Bruce, D., Fletcher, J. M., ... \& Eisenberg, H. M. (1993). Cognition in relation to magnetic resonance imaging in headinjured children and adolescents. Archives of neurology, 50(9), 897-905.

Lighthall, J. W. (1988). Controlled cortical impact: a new experimental brain injury model. Journal of neurotrauma, 5(1), 1-15.

Lindner, M. D., Plone, M. A., Cain, C. K., Frydel, B., Francis, J. M., Emerich, D. F., \& Sutton, R. L. (1998). Dissociable long-term cognitive deficits after frontal versus sensorimotor cortical contusions. Journal of neurotrauma, 15(3), 199-216. 
Locher, C., Koechlin, H., Zion, S. R., Werner, C., Pine, D. S., Kirsch, I., ... \& Kossowsky, J. (2017). Efficacy and safety of selective serotonin reuptake inhibitors, serotoninnorepinephrine reuptake inhibitors, and placebo for common psychiatric disorders among children and adolescents: a systematic review and meta-analysis. Jama Psychiatry, 74(10), 1011-1020.

Long, J. B., Bentley, T. L., Wessner, K. A., Cerone, C., Sweeney, S., \& Bauman, R. A. (2009). Blast overpressure in rats: recreating a battlefield injury in the laboratory. Journal of neurotrauma, 26(6), 827-840.

Maas, A. I., Stocchetti, N., \& Bullock, R. (2008). Moderate and severe traumatic brain injury in adults. The Lancet Neurology, 7(8), 728-741.

Martens, K. M., Vonder Haar, C., Hutsell, B. A., \& Hoane, M. R. (2012). A discrimination task used as a novel method of testing decision-making behavior following traumatic brain injury Journal of Neurotrauma, 29, 2505-2512.

Menon, D. K., Schwab, K., Wright, D. W., \& Maas, A. I. (2010). Position statement: definition of traumatic brain injury. Archives of physical medicine and rehabilitation, 91(11), 16371640.

Menon, V., Adleman, N. E., White, C. D., Glover, G. H., \& Reiss, A. L. (2001). Error-related brain activation during a Go/NoGo response inhibition task. Human brain mapping, 12(3), 131-143.

Mayer, A. R., Mannell, M. V., Ling, J., Elgie, R., Gasparovic, C., Phillips, J. P., ... \& Yeo, R. A. (2009). Auditory orienting and inhibition of return in mild traumatic brain injury: a FMRI study. Human brain mapping, 30(12), 4152-4166. 
Mcintosh, T. K., Noble, L., Andrews, B., \& Faden, A. I. (1987). Traumatic brain injury in the rat: characterization of a midline fluid-percussion model. Central Nervous System Trauma, 4(2), 119-134.

Milner, B. (1963). Effects of different brain lesions on card sorting: The role of the frontal lobes. Archives of neurology, 9(1), 90-100.

Miremami, J. D., Talauliker, P. M., Harrison, J. L., \& Lifshitz, J. (2014). Neuropathology in sensory, but not motor, brainstem nuclei of the rat whisker circuit after diffuse brain injury. Somatosensory \& motor research, 31(3), 127-135.

Morales, D. M., Marklund, N., Lebold, D., Thompson, H. J., Pitkanen, A., Maxwell, W. L., ... \& Graham, D. I. (2005). Experimental models of traumatic brain injury: do we really need to build a better mousetrap?. Neuroscience, 136(4), 971-989.

Moreno, J. A., Arango Lasprilla, J. C., Gan, C., \& McKerral, M. (2013). Sexuality after traumatic brain injury: a critical review. NeuroRehabilitation, 32(1), 69-85.

Moritz, K. E., Geeck, K., Underly, R. G., Searles, M., \& Smith, J. S. (2014). Post-operative environmental enrichment improves spatial and motor deficits but may not ameliorate anxiety-or depression-like symptoms in rats following traumatic brain injury. Restorative neurology and neuroscience, 32(5), 701-716.

Namjoshi, D. R., Cheng, W. H., McInnes, K. A., Martens, K. M., Carr, M., Wilkinson, A., ... \& Wellington, C. L. (2014). Merging pathology with biomechanics using CHIMERA (Closed-Head Impact Model of Engineered Rotational Acceleration): a novel, surgeryfree model of traumatic brain injury. Molecular neurodegeneration, 9(1), 55. 
Nguyen, R., Fiest, K. M., McChesney, J., Kwon, C. S., Jette, N., Frolkis, A. D., ... \& Pringsheim, T. (2016). The international incidence of traumatic brain injury: a systematic review and meta-analysis. Canadian journal of neurological sciences, 43(6), 774-785.

Numan, R., Seifert, A. R., \& Lubar, J. F. (1975). Effects of medio-cortical frontal lesions on DRL performance in the rat. Physiological Psychology, 3(4), 390-394.

Paradiso, S., Chemerinski, E., Yazici, K. M., Tartaro, A., \& Robinson, R. G. (1999). Frontal lobe syndrome reassessed: comparison of patients with lateral or medial frontal brain damage. Journal of Neurology, Neurosurgery \& Psychiatry, 67(5), 664-667.

Passetti, F., Chudasama, Y., \& Robbins, T. W. (2002). The frontal cortex of the rat and visual attentional performance: dissociable functions of distinct medial prefrontal subregions. Cerebral cortex, 12(12), 1254-1268.

Paxinos, G., \& Watson, C. (2009). The rat brain in stereotaxic coordinates (Vol. 6). London: Academic Press.

Ponsford, J., Draper, K., \& Schönberger, M. (2008). Functional outcome 10 years after traumatic brain injury: its relationship with demographic, injury severity, and cognitive and emotional status. Journal of the International Neuropsychological Society, 14(2), 233242.

Rostami, E., Davidsson, J., Ng, K. C., Lu, J., Gyorgy, A., Wingo, D., ... \& Risling, M. (2012). A model for mild traumatic brain injury that induces limited transient memory impairment and increased levels of axon related serum biomarkers. Frontiers in neurology, 3, 115.

Rosnick, C. B., Wetherell, J. L., White, K. S., Andreescu, C., Dixon, D., \& Lenze, E. J. (2016). Cognitive-behavioral therapy augmentation of SSRI reduces cortisol levels in older adults 
with generalized anxiety disorder: A randomized clinical trial. Journal of consulting and clinical psychology, 84(4), 345.

Scott, T. L., \& Vonder Haar, C. (2019). Frontal brain injury chronically impairs timing behavior in rats. Behavioural brain research, 356, 408-414.

Schoenbaum, G., \& Roesch, M. (2005). Orbitofrontal cortex, associative learning, and expectancies. Neuron, 47(5), 633-636.

Schönfeld, L. M., Jahanshahi, A., Lemmens, E., Schipper, S., Dooley, D., Joosten, E., ... \& Hendrix, S. (2017). Long-Term Motor Deficits after Controlled Cortical Impact in Rats Can Be Detected by Fine Motor Skill Tests but Not by Automated Gait Analysis. Journal of neurotrauma, 34(2), 505-516.

Silverberg, N. D., Crane, P. K., Dams-O'Connor, K., Holdnack, J., Ivins, B. J., Lange, R. T., ... \& Iverson, G. L. (2017). Developing a cognition endpoint for traumatic brain injury clinical trials. Journal of neurotrauma, 34(2), 363-371.

Sokolowski, J. D., \& Salamone, J. D. (1994). Effects of dopamine depletions in the medial prefrontal cortex on DRL performance and motor activity in the rat. Brain research, 642(1-2), 20-28.

Soldatovic-Stajic, B., Misic-Pavkov, G., Bozic, K., Novovic, Z., \& Gajic, Z. (2014).

Neuropsychological and neurophysiological evaluation of cognitive deficits related to the severity of traumatic brain injury. Eur Rev Med Pharmacol Sci, 18(11), 1632-7.

Stuss, D. T., Levine, B., Alexander, M. P., Hong, J., Palumbo, C., Hamer, L., ... \& Izukawa, D. (2000). Wisconsin Card Sorting Test performance in patients with focal frontal and posterior brain damage: effects of lesion location and test structure on separable cognitive processes. Neuropsychologia, 38(4), 388-402. 
Tait, S. D., Chase, A. E., \& Brown, J. V. (2014). Attentional set-shifting in rodents: a review of behavioural methods and pharmacological results. Current pharmaceutical design, 20(31), 5046-5059.

Teasdale, G., \& Jennett, B. (1974). Assessment of coma and impaired consciousness: a practical scale. The Lancet, 304(7872), 81-84.

Uslaner, J. M., \& Robinson, T. E. (2006). Subthalamic nucleus lesions increase impulsive action and decrease impulsive choice- mediation by enhanced incentive motivation? European Journal of Neuroscience, 24(8), 2345-2354.

Vonder Haar, C., Anderson, G. D., \& Hoane, M. R. (2011). Continuous nicotinamide administration improves behavioral recovery and reduces lesion size following bilateral frontal controlled cortical impact injury. Behavioural brain research, 224(2), 311-317.

Vonder Haar, C., \& Winstanley, C. A. (2016). Minor functional deficits in basic response patterns for reinforcement after frontal traumatic brain Injury in rats. Journal of neurotrauma, 33(20), 1892-1900

Vonder Haar, C., Lam, F. C., Adams, W. K., Riparip, L. K., Kaur, S., Muthukrishna, M., ... \& Winstanley, C. A. (2016). Frontal traumatic brain injury in rats causes long-lasting impairments in impulse control that are differentially sensitive to pharmacotherapeutics and associated with chronic neuroinflammation. ACS chemical neuroscience, 7(11), 1531-1542.

Vonder Haar, C., Maass, W. R., Jacobs, E. A., \& Hoane, M. R. (2014). Deficits in discrimination after experimental frontal brain injury are mediated by motivation and can be improved by nicotinamide administration. Journal of neurotrauma, 31(20), 1711-1720. 
Vonder Haar, C., Smith, T. R., French, E. J., Martens, K. M., Jacobs, E. A., \& Hoane, M. R. (2014). Simple tone discriminations are disrupted following experimental frontal traumatic brain injury in rats. Brain injury, 28(2), 235-243.

Verbruggen, F., Aron, A. R., Band, G. P., Beste, C., Bissett, P. G., Brockett, A. T., ... \& Colzato, L. S. (2019). A consensus guide to capturing the ability to inhibit actions and impulsive behaviors in the stop-signal task. Elife, 8, e46323.

Walker, W. C., \& Pickett, T. C. (2007). Motor impairment after severe traumatic brain injury: a longitudinal multicenter study. Journal of rehabilitation research and development, $44(7), 975$.

Whitnall, L., McMillan, T. M., Murray, G. D., \& Teasdale, G. M. (2006). Disability in young people and adults after head injury: 5-7 year follow up of a prospective cohort study. Journal of Neurology, Neurosurgery \& Psychiatry, 77(5), 640-645.

Wilson, M. P., \& Keller, F. S. (1953). On the selective reinforcement of spaced responses. Journal of Comparative and Physiological Psychology, 46(3), 190.

Wood, R. L., \& Thomas, R. H. (2013). Impulsive and episodic disorders of aggressive behaviour following traumatic brain injury. Brain injury, 27(3), 253-261. 